Published in final edited form as:

J Chem Inf Model. 2019 July 22; 59(7): 3291-3304. doi:10.1021/acs.jcim.9b00334.

\title{
AGL-Score: Algebraic Graph Learning Score for Protein-Ligand Binding Scoring, Ranking, Docking, and Screening
}

\author{
Duc Duy Nguyen ${ }^{\dagger}$ Guo-Wei Wei ${ }^{\dagger, \ddagger, \uparrow}$ \\ †Department of Mathematics, Michigan State University, MI 48824, USA \\ ‡Department of Biochemistry and Molecular Biology, Michigan State University, MI 48824, USA \\ "Department of Electrical and Computer Engineering, Michigan State University, MI 48824, USA
}

\begin{abstract}
Although algebraic graph theory based models have been widely applied in physical modeling and molecular studies, they are typically incompetent in the analysis and prediction of biomolecular properties, confirming the common belief that "one cannot hear the shape of a drum". A new development on this century old issue about spectrum-geometry relationship is provided. Novel algebraic graph learning score (AGL-Score) models are proposed to encode high-dimensional physical and biological information into intrinsically low-dimensional representations. The proposed AGL-Score models employ multiscale weighted colored subgraphs to describe crucial molecular and biomolecular interactions in terms of graph invariants derived from graph Laplacian, its pseudo-inverse, and adjacency matrices. Additionally, AGL-Score models are integrated with an advanced machine learning algorithm to predict biomolecular macroscopic properties from the low-dimensional graph representation of biomolecular structures. The proposed AGL-Score models are extensively validated for their scoring power, ranking power, docking power, and screening power via a number of benchmark datasets, namely CASF-2007, CASF-2013, and CASF-2016. Numerical results indicate that the proposed AGL-Score models are able to outperform other state-of-the-art scoring functions in protein-ligand binding scoring, ranking, docking, and screening. This study indicates that machine learning methods are powerful tools for molecular docking and virtual screening. It also indicates that spectral geometry or spectral graph theory has the ability to infer geometric properties.
\end{abstract}

weig@msu.edu.

Supporting Information Available

See Suppl4.pdf for the cross-validation performance of AGL models on the training data set of CASF-2007 benchmark, some additional results on ranking power assessments, and discrepancy between CASF-2016 test set and PDBbind v2016 core set.

Availability

The algebraic graph learning score (AGL-Score) model is implemented on our own hosted server at https://weilab.math.msu.edu/AGLScore. Users just need to provide the ligand 3D structure in sdf or mol2 format and protein 3D structure in pdb format for the binding affinity prediction. If the calculation needs a longer time than usual, the user can provide email for the job completion notice. Training sets for the docking power assessment and additional training sets for the screening power assessment are also available for download at https://weilab.math.msu.edu/AGL-Score. 


\section{Introduction}

Graph theory is a prime subject of discrete mathematics that concerns graphs as mathematical structures for modeling pairwise relations between vertices, nodes, or points. Such pairwise relations define graph edges. There are many different graph theories, such as geometric graph theory, algebraic graph theory, and topological graph theory. Geometric graphs admit geometric objects as graph nodes or vertices. Algebraic graph theory, particularly spectral graph theory, studies the algebraic connectivity via characteristic polynomial, eigenvalues, and eigenvectors of matrices associated with graphs, such as adjacency matrix or Laplacian matrix. Topological graph theory concerns the embeddings and immersions of graphs, and the association of graphs with topological spaces, such as abstract simplicial complexes. Mathematically, graphs are useful tools in geometry and certain parts of topology such as knot theory and algebraic topology.

Like topology, graph theory also emphasizes connectivity. The geometric connectivity of a graph refers to pairwise relations among graph nodes and is often analyzed by "topological index", 1,2 contact map ${ }^{3,4}$ and graph centrality. ${ }^{5-7}$ The algebraic connectivity of a graph refers to the second-smallest eigenvalue of the Laplacian matrix of the graph and is also known as Fiedler value or Fiedler eigenvalue, which has many applications, including the stability analysis of dynamical systems. ${ }^{8}$ In contrast, topological connectivity refers to the connectedness of the entire system rather than pairwise ones as in the geometric graph theory. Topological connectivity is an important property for distinguishing topological spaces.

Over a century ago, Hermann Weyl investigated whether geometric properties of bounded domain could be determined from the eigenvalues of the Laplace operator on the domain. This question was phrased as "Can one hear the shape of a drum?" by Mark Kac. ${ }^{9}$ An interesting question is: Can eigenvalues describe protein-ligand binding?

Graph theory has been widely applied in physical, chemical, biological, social, linguistic, computer and information sciences. Many practical problems can be represented and analyzed by graphs. In chemistry and biology, a graph makes a natural model for a molecule, where graph vertices represent atoms and graph edges represent possible bonds. Graphs have been widely used in chemical analysis ${ }^{10-12}$ and biomolecular modeling, ${ }^{13}$ including normal mode analysis (NMA) ${ }^{14-17}$ and elastic network model (ENM) $)^{3,18-22}$ for modeling protein flexibility and long-time dynamics. Some of the most popular ENMs are Gaussian network model (GNM) $)^{3,19,23}$ and anisotropic network model (ANM) ${ }^{20}$ In these methods, the diagonalization of the interaction Laplacian matrix is a required procedure to analyze protein flexibility, which has the computational complexity of $\mathrm{O}\left(N^{3}\right)$ with $\mathrm{N}$ being the number of matrix elements. Graph theory has also been used to represent the structures of molecules and biomolecules, resulting in a popular approach for chemical datasets ${ }^{2,10,11,24-26}$ and biomolecular datasets $3,27-32$ in the past few decades.

Graph theories, especially geometric graph theories, are relatively intuitive and easy to use. Indeed, a great portion of graph theory based study in molecular and biomolecular systems was qualitative and descriptive. Despite the intensive effort in the past, graph theory based 
quantitative methods are often not as competitive as other quantitative approaches in the analysis and prediction of biomolecular properties from massive and diverse datasets. For example, graph-signature based prediction of protein stability changes upon mutation ${ }^{33}$ was not as accurate as some other methods. ${ }^{34-36}$ Additionally, the average Pearson correlation coefficients in protein B-factor predictions using spectral graph theory based Gaussian network model (GNM) were less than 0.6 in all of three datasets. ${ }^{37}$ These situations may be due to the following reasons. Firstly, most graph theory based models do not distinguish different chemical element types in a molecule or biomolecule, which leads to a severe loss of critical chemical and biological information. Secondly, in many molecular graphs, edges are used to represent covalent bonds, while non-covalent interactions are often ignored, which underrepresents the physical interactions of many biomolecular datasets. Finally, many graph-based models approximate the distance between a pair of atoms by the number of covalent bonds between them, which leads to a major error in describing their interaction strength.

In the past few years, we have developed a number of graph theory approaches to address the aforementioned problems. For example, weighted graphs were proposed in terms of the flexibility-rigidity index (FRI) to represent graph edges by radial basis functions. ${ }^{38-41}$ Physically, we assume that protein interactions, including those with its environment, fully determine its structure at the equilibrium. Protein structure and its environment, in turn, fully determine protein flexibility and function. As a consequence, one does not need to invoke a high-dimensional model that is subject to modeling errors to analyze protein flexibility and function when the native structure of the protein and its environment are known.

Mathematically, our approach assumes a complete graph while it weights the importance of graph edges by scaling their Euclidean distances in radial basis functions so that the nearest neighbors in the sense of the Euclidean metric have the strongest edges. Additionally, multiscale FRI is a multigraph approach which is permitted to have multiple edges. ${ }^{40,42}$ Similar to persistent homology, ${ }^{43,44}$ this multi-edge technique allows a given molecular graph to be analyzed in multiscale, capturing the multiscale interactions in macromolecules. ${ }^{40}$ Graph coloring, or more generally, graph labeling, is an important graph theory technique for graph vertices or edges to be treated differently. This method enables the encoding of chemical and biological information into molecular graphs. ${ }^{45,46}$ Subgraphs constructed from vertex-labeled graphs and edge-labeled graphs give rise to powerful graph representations of intermolecular and intramolecular interactions, such as hydrogen bonds, electrostatics, van der Waals interactions, hydrophilicity, hydrophobicity etc. ${ }^{45,46}$ Our multiscale weighted colored graph is over $40 \%$ more accurate than GNM in protein B-factor predictions ${ }^{46}$.

The importance of protein-ligand binding for in living organisms cannot be overstated. A wide variety of biological processes, such transmitter-mediated signal transduction, hormone and growth factor regulated metabolic pathways, and stimulus-initiated gene expression, enzyme production, and cell secretion, are triggered by ligand-receptor agonist binding. Therefore, the understanding of protein-ligand interactions is a central issue in biochemistry, biophysics and molecular biology. It is commonly believed that protein-ligand binding involves synergistic protein-ligand corporation, molecular recognition, and conformational changes for both protein and ligand. 
Various scoring functions (SFs) have been developed for understanding protein-ligand binding. Among them, physics based SFs uniquely offer the mechanistic understanding and do not depend on existing data. Empirical SFs use physical submodels to fit existing data. 47-49 Knowledge-based SFs take the advantage of available protein-ligand binding datasets and can be used without further training. ${ }^{50-52}$ Finally, machine learning-based SFs are datadriven and their performance strongly depends on the training set, in addition to their descriptors and machine learning algorithms. ${ }^{53-58}$ On the positive side, machine learningbased SFs can easily handle large and diverse datasets, as well as nonlinear correlations in the data. They often turn out as the winners for many standard benchmarks $36,45,53,57,58$ and community-wide competitions. ${ }^{59}$

Notably, RF-Score was the first machine learning-based SF that impressively outperformed other SFs in $2010 .{ }^{53}$ Since then, there has been much skepticism about machine learning based SFs. For example, Gabel et al have shown that RF-Score is unable to enrich virtual screening hit lists in true actives upon docking experiments of 10 reference DUDE datasets. ${ }^{60}$ This comes with no surprise. All machine learning-based scoring functions are datadriven methods and do not work without structural and/or sequence similarity in training and prediction datasets as shown by Li and Yang. ${ }^{61}$ It can be hard to decide what training set should be used, while Kramer et al argued that leave-cluster-out cross-validation is appropriate for scoring functions derived from diverse protein data sets. ${ }^{62}$ Recently, Wang and Zhang have generated their own training sets ${ }^{1}$ to show that machine learning models can do very well in docking and screening tests. ${ }^{58}$ It is highly important to design common benchmarks ${ }^{63-66}$ and/or blind grand challenges so that various scoring functions can be assessed on an equal footing without bias and prejudice.

Recently, we have developed various machine learning-based SFs using one of three types of descriptors, namely physics-based descriptors which consist of electrostatics binding free energies and atomic Coulombic and van der Waals interactions, ${ }^{57}$ geometric graph theorybased descriptors, ${ }^{45}$ and algebraic topology-based descriptors. ${ }^{36}$ Geometric graph theory and algebraic topology-based predictions of free energies and their rankings (Kendall's tau) were ranked 1st for Set 1 (Stage 2) of D3R Grand Challenge 2 and for 10 of a total of 26 contests in D3R Grand Challenge 3. ${ }^{59}$ Conceptually, our approaches are built upon the fundamental hypothesis that the intrinsic physics of interest lies in low-dimensional subspaces or manifolds embedded in a high-dimensional data space. While the hypothesis is quite wellknown in the manifold learning field, the major challenge is how to encode crucial physical information contained in a high-dimensional space into the desirable low-dimensional representation of molecules, biomolecules and their complexes. Our multiscale weighted colored subgraphs address this challenge. The major advantages of our multiscale weighted colored subgraph approach are its low-dimensionality, simplicity, and robustness. For example, the only required data inputs for the binding affinity prediction are atomic names and coordinates. Indeed, it bypasses complicated data processing and parametrization. It does not need any molecular mechanical (MM) force fields, namely, charges, polarization assignments, bond lengths and angles, van der Waals well depths, dielectric constants,

\footnotetext{
${ }^{1}$ Unfortunately, these useful training sets are not publicly available.
} 
surface tension, electronegativity, etc. As such, it avoids errors associated with the parametrization. In fact, our geometric graph theory approach is also simpler than our algebraic topology approach mathematically and computationally, while it performs as well as our topological approach. ${ }^{67}$

The objective of the present work is to develop multiscale weighted labeled algebraic subgraphs for representing protein-ligand interactions. For a given protein-ligand complex, there are many ways to construct corresponding algebraic (sub)graphs. Three most commonly used algebraic graphs are graph Laplacian matrix, its pseudo-inverse, and adjacency matrices. The eigenvalues and eigenvectors computed from these matrices can be used to describe molecules, biomolecules and their interactions in many different ways. We examine a few common approaches in this work. The resulting method, called algebraic graph learning score ( AGL-Score), is critically assessed on its protein-ligand binding scoring power, ranking power, docking power, and screening power with a variety of benchmark datasets.

\section{Methods and Algorithms}

\subsection{Multiscale weighted labeled geometric subgraphs}

We propose to develop systematical, scalable, accurate graph theory descriptors of proteinligand binding interactions from massive and diverse datasets. However, the proposed method can be applied to other problems such as the predictions of toxicity, solubility, solvation, partition coefficient, mutation-induced protein folding stability change, and protein- nucleic acid interactions. In the present work, we target pairwise non-covalent interactions in our subgraph theory description. For a given dataset, we first perform a statistical analysis to identify a set of commonly occurring chemical element types, say $\mathscr{C}=\{\mathrm{H}, \mathrm{C}, \mathrm{N}, \mathrm{O}, \mathrm{S}, \mathrm{P}, \mathrm{F}, \mathrm{Cl}, \mathrm{Br}, \cdots\}$. For a given molecule or biomolecule in the dataset, let us denote

$$
\mathscr{V}=\left\{\left(\mathbf{r}_{j}, \alpha_{j}\right) \mid \mathbf{r}_{j} \in \mathbb{R}^{3} ; \alpha_{j} \in \mathscr{C} ; j=1,2, \ldots, N\right\}
$$

a subset of $N$ atoms (i.e., subgraph vertices) that are members of $C$. Note that the $i$ atom is labeled both by its element type $a_{j}$ and its position $\mathbf{r}_{j}$. The classification of atoms into chemical element types is a graph coloring, which is important for encoding different types of interactions and gives rise to a basis for the collective coarse-grained description of the dataset.

We assume that all the pairwise non-covalent interactions between element types $C_{k}$ and $\mathscr{C}_{k^{\prime}}$ in a molecule or molecular complex can be represented by fast-decay weight functions

$$
\mathscr{E}=\left\{\Phi\left(\| \mathbf{r}_{i}-\mathbf{r}_{j}|| ; \eta_{k k^{\prime}}\right) \mid \alpha_{i}=\mathscr{C}_{k}, \alpha_{j}=\mathscr{C}_{k^{\prime}} ; i, j=1,2, \ldots, N ;\left\|\mathbf{r}_{i}-\mathbf{r}_{j}\right\|>r_{i}+r_{j}+\sigma\right\}
$$


where $\left\|\mathbf{r}_{i}-\mathbf{r}_{j}\right\|$ is the Euclidean distance between the $i^{\text {th }}$ and $j^{\text {th }}$ atoms, $r_{i}$ and $r_{j}$ are the atomic radii of $i^{\text {th }}$ and $j^{\text {th }}$ atoms, respectively and $\sigma$ is the mean value of the standard deviations of $r_{j}$ and $r_{k}$ in the dataset. The distance constraint $\left(\| \mathbf{r}_{i}-\mathbf{r}_{j}||>r_{i}+r_{j}+\sigma\right)$ excludes covalent interactions. Here $\eta_{k k^{\prime}}$ is a characteristic distance between the atoms, and $\Phi$ is a subgraph weight and is chosen to have the following properties: ${ }^{39}$

$$
\begin{gathered}
\Phi\left(\left\|\mathbf{r}_{i}-\mathbf{r}_{j}\right\| ; \eta_{k k^{\prime}}\right)=1, \text { as }\left\|\mathbf{r}_{i}-\mathbf{r}_{j}\right\| \rightarrow 0 \text { and } \\
\Phi\left(\left\|\mathbf{r}_{i}-\mathbf{r}_{j}\right\| ; \eta_{k k^{\prime}}\right)=0 \text { as }\left\|\mathbf{r}_{i}-\mathbf{r}_{j}\right\| \rightarrow \infty, \alpha_{i}=\mathscr{C}_{k}, \alpha_{j}=\mathscr{C}_{k^{\prime}}
\end{gathered}
$$

Although most radial basis functions can be used, generalized exponential functions and generalized Lorentz functions were shown to work very well for biomolecules. ${ }^{39} \mathrm{We}$, therefore, have a weighted colored subgraph $G(\mathscr{V}, \mathscr{E})$. To construct element-level collective molecular descriptors, we propose the multiscale weighted colored subgraph rigidity between $k$ th element type $C_{k}$ and $k^{\prime}$ th element type $\mathscr{C}_{k^{\prime}}$

$$
\begin{aligned}
& \mathrm{RI}^{G}\left(\eta_{k k^{\prime}}\right)=\sum_{i} \mu_{i}^{G}\left(\eta_{k k^{\prime}}\right)=\sum_{i} \sum_{j} \Phi\left(\left\|\mathbf{r}_{i}-\mathbf{r}_{j}\right\| ; \eta_{k k^{\prime}}\right), \alpha_{i}=\mathscr{C}_{k}, \alpha_{j}=\mathscr{C}_{k^{\prime}},\left\|\mathbf{r}_{i}-\mathbf{r}_{j}\right\|>r_{i}+r_{j} \\
& +\sigma
\end{aligned}
$$

where $\mu_{i}^{G}\left(\eta_{k k^{\prime}}\right)$ is a geometric subgraph centrality for the $\dot{t}$ h atom, which offers accurate protein B-factors predictions. ${ }^{46}$ The physical interpretation of Eq. (5) is straightforward the summation over $\mu_{i}^{G}\left(\eta_{k k^{\prime}}\right)$ in Eq.(5) leads to the total interaction strength for the selected pair of element types $\mathscr{C}_{k}$ and $\mathscr{C}_{k^{\prime}}$, which provides the element-level coarse-grained description of molecular level properties. The above formulation is a generalization of the successful bipartite subgraph used in our earlier predictions of protein-ligand binding affinities and free energy ranking. ${ }^{45}$ For a bipartite subgraph, each of its edges connects one atom in the protein and another atom in the ligand. The graph coloring, i.e., element specific descriptions, and subgraph weight are designed to capture hydrogen bonds, polarization, electrostatics, van der Waals interactions, hydrophilicity, hydrophobicity, etc.

The different selections of characteristic distance $\eta_{k k^{\prime}}$ give rise to a multiscale description of intermolecular and intramolecular interactions. By appropriate selections of element combinations $k$ and $k^{\prime}$, the characteristic distance $\eta_{k k^{\prime}}$, and subgraph weight $\phi$, we systematically construct a family of collective, scalable, multiscale graph-based molecular 
and biomolecular descriptors. The proposed multiscale weighted colored subgraph rigidity is simple and robust - the only required data input is atomic names and coordinates. Indeed, it bypasses complicated data processing, parametrization, and molecular mechanical force fields, such as charges, high-order polarizations, van der Waals well depths, dielectric constants, surface tensions, and electronegativity, and their associated errors in many physical models. Consequently, our graph theory approaches are very fast. ${ }^{39}$ Our fast algorithm has the computational complexity of $\mathrm{O}(N)$ and is able to predict B-factors for acarbons of an HIV virus capsid (313 236 residues) in less than 30 seconds on a single processor. $^{39}$

\subsection{Multiscale weighted labeled algebraic subgraphs}

Our earlier work has demonstrated how to construct powerful geometric graph descriptors for analyzing and predicting biomolecular datasets. Mathematically, it is extremely interesting to understand whether there exist equally powerful algebraic graph or spectral graph formulations. Biologically, it is important to develop alternative graph tools for describing molecules, biomolecules and their interactions since each method has its own advantages and potentials. For a given subgraph, its matrix representations provide a straightforward description of the interaction between subgraph elements, which can be easily expressed by matrices. Two of the most important matrices are the Laplacian matrix and the adjacency matrix.

Multiscale weighted labeled Laplacian matrix - We consider a subgraph $G_{k k^{\prime}}$ for each pair of element types, $C_{k}$ and $C_{k^{\prime}}$, and propose an element-level weighted labeled Laplacian matrix $L\left(\eta_{k k^{\prime}}\right)$ with elements

$$
L_{i j}\left(\eta_{k k^{\prime}}\right)=\left\{\begin{array}{cc}
-\Phi\left(\left\|\mathbf{r}_{i}-\mathbf{r}_{j}\right\| ; \eta_{k k^{\prime}}\right) & \begin{array}{c}
\text { if } i \neq j, \alpha_{i}=\mathscr{C}_{k}, \alpha_{j}=\mathscr{C}_{k^{\prime}} \\
\\
\text { and }\left\|\mathbf{r}_{i}-\mathbf{r}_{j}\right\|>r_{i}+r_{j}+\sigma
\end{array} \\
-\sum_{j} L_{i j} & \text { if } i=j .
\end{array}\right.
$$

Mathematically, our element-level weighted labeled Laplacian matrix is symmetric, diagonally dominant and positive-semidefinite, and thus all of its eigenvalues are nonnegative. Since every row sum or column sum of $L\left(\eta_{k k^{\prime}}\right)$ is zero, the first eigenvalue value is zero. There can be more than one zero eigenvalue and the number of zero eigenvalues of the Laplacian is the rank of the zero-dimensional topological invariant, reflecting the number of the connected components in the graph. The first non-zero eigenvalue of $L\left(\eta_{k k^{\prime}}\right)$ is the socalled algebraic connectivity (or Fiedler value) of $G_{k k^{\prime}}$. It is interesting to note a certain connection between geometric graph formulation and algebraic graph matrix:

$$
\mathrm{RI}^{G}\left(\eta_{k k^{\prime}}\right)=\operatorname{Tr} L\left(\eta_{k k^{\prime}}\right)
$$


where $\operatorname{Tr}$ is the trace. Denote $\lambda_{j}^{L}, j=1,2, \cdots$ and $\mathbf{u}_{j}^{L}, j=1,2, \cdots$ the eigenvalues and eigenvectors of $L\left(\eta_{k k^{\prime}}\right)$. We define an atomic descriptor for the $i$ th atom $\left(\mathbf{r}_{i}, \alpha_{i}=\mathscr{C}_{k}\right)$ :

$$
\mu_{i}^{L}\left(\eta_{k k^{\prime}}\right)=\sum_{l}\left(\lambda_{l}^{L}\right)^{-1}\left[\mathbf{u}_{l}^{L}\left(\mathbf{u}_{l}^{L}\right)^{T}\right]_{i i}
$$

where $T$ is the transpose. We further propose element-level weighted labeled Laplacian matrix based molecular descriptors

$$
\mathrm{RI}^{L}\left(\eta_{k k^{\prime}}\right)=\sum_{i} \mu_{i}^{L}\left(\eta_{k k^{\prime}}\right)
$$

Note that $\mu_{i}^{L}\left(\eta_{k k^{\prime}}\right)$ is a weight subgraph generalization of $\mathrm{GNM}^{3}$ or a subgraph generalization of our earlier generalized multiscale FRI. ${ }^{42}$ Therefore, $\mu_{i}^{L}\left(\eta_{k k^{\prime}}\right)$ can be used to represent atomic properties, such as protein B-factors. Additionally, we can construct a set of elementlevel weighted labeled Laplacian matrix based molecular descriptors by the statistics of $\mu_{i}^{L}\left(\eta_{k k^{\prime}}\right)$, i.e., sum, mean, maximum, minimum and standard deviation of $\mu_{i}^{L}\left(\eta_{k k^{\prime}}\right)$.

Alternatively, we can directly construct another set of element-level weighted labeled Laplacian matrix based molecular descriptors by the statistics of nontrivial eigenvalues $\left\{\lambda_{j}^{L}\right\}_{j=2,3, \ldots}$. In this case, the Fiedler value is included as the minimum. The performances of these two sets of molecular descriptors based constructed from element-level weighted labeled subgraph Laplacian matrix will be examined and compared.

Multiscale weighted labeled adjacency matrix-The element-level weighted labeled adjacency matrix is equally important and can be easily constructed for subgraph $G_{k k^{\prime}}$ by

$$
A_{i j}\left(\eta_{k k^{\prime}}\right)= \begin{cases}\Phi\left(\left\|\mathbf{r}_{i}-\mathbf{r}_{j}\right\| ; \eta_{k k^{\prime}}\right) & \text { if } i \neq j, \alpha_{i}=\mathscr{C}_{k}, \alpha_{j}=\mathscr{C}_{k^{\prime}} \\ \text { and }\left\|\mathbf{r}_{i}-\mathbf{r}_{j}\right\|>r_{i}+r_{j}+\sigma\end{cases}
$$

Mathematically, adjacency matrix $A\left(\eta_{k k^{\prime}}\right)$ is a symmetric non-negative matrix and it contains the same amount of information as the corresponding Laplacian matrix, although its eigenvalues $\lambda_{j}^{A}, j=1,2, \cdots$ and eigenvectors $\mathbf{u}_{j}^{A}, j=1,2, \cdots$ behave very differently from those of corresponding Laplacian matrix. Fig. 1 illustrates the Laplacian and adjacency matrices for the weighted colored subgraph $G_{\mathrm{NO}}$ in cellocidin molecule $\left(\mathrm{C}_{4} \mathrm{H}_{4} \mathrm{~N}_{2} \mathrm{O}_{2}\right)$. The spectrum of the proposed element-level weighted colored adjacency matrix is real. For each eigenvalue, its opposite is also an eigenvalue. Therefore, only positive eigenvalues will be used in our description. The Perron-Frobenius theorem states that the greatest eigenvalue, 
ie., the spectral radius $\rho(A)$, is bounded above by the maximal diagonal element of the corresponding Laplacian matrix $\min _{i} \sum_{j} A_{i j} \leq \rho(A) \leq \max _{i} \sum_{j} A_{i j}$. The values of Laplacian matrix elements depend on the scale parameter $\eta_{k k}$ ' and have many zeros at a characteristic scale parameter for hydrogen bonds or van der Waals interactions. However characteristic scale for electrostatic and hydrophobic interactions can be very large. ${ }^{68}$ In such as case, spectral radius $\max _{i} \sum_{j} A_{i j} \approx n-1$, with $n$ being the number of atoms in the subgraph $G_{k k^{\prime}}$. Assume that all eigenvalues and eigenvectors of $A_{i j}\left(\eta_{k k^{\prime}}\right)$ are given by $\left\{\lambda_{j}^{A}\right\}$ and $\left\{\mathbf{u}_{j}^{A}\right\}$, respectively. In the present work, we use a set of statistical values, namely, the sum of all positive eigenvalues, the mean of all positive eigenvalues, the largest (i.e., the principal) and the smallest positive eigenvalues, and the standard deviation of all positive eigenvalues as element-level molecular descriptors of molecules, biomolecules and their interactions.

In principle, we can also construct atomic descriptors from $\left\{\lambda_{j}^{A}\right\}$ and $\left\{\mathbf{u}_{j}^{A}\right\}$. Let us define a square matrix $Q$ whose columns are n linearly independent eigenvectors of $A: Q=\left[\mathbf{u}_{1}^{A} \mathbf{u}_{2}^{A} \cdots \mathbf{u}_{n}^{A}\right]$ and a diagonal matrix $\Lambda$ where each diagonal element $\Lambda_{i i}$ is the eigenvalue associated with the th column of $Q$. Then, a set of atomic descriptors can be obtained as

$$
\mu_{i}^{A}\left(\eta_{k k^{\prime}}\right)=\sum_{j}\left[Q \Lambda Q^{-1}\right]_{i j}
$$

However, the method given in Eq.(10) is not a computationally efficient approach for describing atoms in molecules.

It might appear that the proposed algebraic graph theory-based method depends on eigenvalue analysis, which is normally very expensive. However, there are two facts that make the proposed method computationally efficient. First, only atoms in a small neighborhood of the protein-ligand binding site are involved in matrix constructions. Additionally, the element-specific selections further reduce the number of atoms involved in each matrix construction. As a result, one just needs to deal with many small matrices, rendering an efficient spectral approach for protein-ligand binding affinity analysis.

\subsection{Graph learning}

To predict molecular and biomolecular properties, statistics of eigenvalues generated from the proposed weighted labeled subgraph Laplacian matrix or adjacency matrix will be combined with a machine learning algorithm. We assume the dataset is labeled and the problem is either a classification or a regression. From the machine learning point of view, we employ a supervised learning algorithm involving a training set and a test set. Denote $X_{i}$ the dataset from the ith molecule or molecular complex in the training dataset and let $\mathbf{G}\left(\mathscr{X}_{i} ; \zeta\right)$ be a function that maps the geometric information into suitable graph representations with a set of parameters $\zeta$ consisting of kernel parameters. To set up a machine learning model, we cast the training into a minimization problem, 


$$
\min _{\zeta, \theta} \sum_{i \in I} \mathscr{L}\left(\mathbf{y}_{i}, \mathbf{G}\left(\mathscr{X}_{i} ; \zeta\right) ; \boldsymbol{\theta}\right)
$$

where $\mathscr{L}$ is a scalar loss function to be minimized and $\mathrm{y}_{i}$ is the collection of labels in the training set. Here $\theta$ are the set of machine learning parameters to be optimized and depend on machine learning algorithms chosen. The loss function $\mathscr{L}$ can be chosen according to the nature of the problem, i.e., regression and classification. Many machine learning algorithms, such as random forest, gradient boosting trees, artificial neural networks, and convolutional neural networks, can be employed in conjugation with the present graph descriptors. However, as our goal in the present work is to examine the descriptive power of the proposed algebraic graph features, let us focus on a relatively simple while still powerful machine learning algorithm, gradient boosting trees (GBTs). GBTs are very robust against overfitting $^{35}$ and their performance is quite similar to that of random forest. Figure 2 illustrates the proposed graph learning strategy.

Throughout this work, we choose GradientBoostingRegressor module implemented in the scikit-learn v0.19.1 package with parameters $n$ _estimators $=10000$, max_depth $=7$, min_samples_split $=3$, learning_rate $=0.01$, loss $=1$ s, subsample $=0.3$, and max_features $=$ sqrt. Changes in these parameters do not significantly affect the prediction results.

\section{Datasets and evaluation metrics}

\subsection{Datasets}

In this work, we validate our proposed model against three commonly drug-discovery related benchmark datasets, namely, CASF-2007, ${ }^{63}$ CASF-2013, ${ }^{64}$ and CASF-2016. ${ }^{66}$ These benchmarks are collected in the PDBbind database and have been used to evaluate the general performance of a scoring function on a diverse set of protein-ligand complexes. The statistical information of these datasets is provided in Table 1. There is a wide variety of SFs in the binding affinity prediction and in pose scoring. In general, one can classify them into four groups: ${ }^{69}$ a) Force-field based or physical based SFs; b) Empirical or linear regression based SFs; c) Potential of the mean force (PMF) or knowledge-based SFs; and d) Machine learning based SFs. The present method falls into the last category.

Note that for docking power and screening power assessments, additional data information is given for CASF-200763 and CASF-2013 ${ }^{64,65}$ as described in the next section.

\subsection{Evaluation metrics}

In this work, we assess the general performance of the proposed model by four metrics, namely, scoring power, ranking power, docking power, and screening power. ${ }^{63,65}$ These metrics and their associated datasets are briefly summarized below.

3.2.1 Scoring power-This assessment validates a scoring function's ability to predict binding affinities that have a linear correlation with experimental data. The evaluation metric used in this task is the standard Pearson's correlation coefficient $\left(R_{p}\right)$ given by 


$$
R_{p}=\frac{\sum\left(x_{i}-\bar{x}\right)\left(y_{i}-\bar{y}\right)}{\sqrt{\sum\left(x_{i}-\bar{x}\right)^{2}} \sqrt{\sum\left(y_{i}-\bar{y}\right)^{2}}},
$$

where $x_{i}$ and $y_{i}$ are, respectively, predicted binding affinity and experimental data for the $t$ th complex. Here $\bar{x}$ and $\bar{y}$ are the average of all predicted values and experimental values in the dataset, respectively. In this work, three datasets, CASF-2007, CASF-2013, and CASF-2016, are employed to test AGL-Score's scoring power.

3.2.2 Ranking power-This assessment validates a scoring function's ability to rank binding affinities of proteinligand complexes in each cluster. ${ }^{63,65}$ Both benchmarks CASF-2007 and CASF-2013 have 65 clusters of complexes, and each cluster has three complexes formed by the same protein but with different ligands. In the so-called high-level success measurement, the binding affinities of three complexes in each cluster are to be correctly ranked. While in the so-called low-level success measurement, a scoring function only needs to pick the structure with the highest binding affinity. The ranking power is evaluated by the percentage of successful identifications in a given benchmark.

In fact, the ranking power metric could be improved. Currently, it only counts the correct order of binding affinities of three native ligands for each target receptor in the core set. Also, it may not reflect the realistic setting of a real virtual screening process, in which a considerable number of ligands can bind to the same target. Additionally, more robust evaluation metrics, such as Kendall's tau or Spearman correlation coefficient, might be used.

3.2.3 Docking power-This assessment validates a scoring function's ability to identify the "native" pose from docking software generated poses. ${ }^{63}$ In the benchmark, a pose is considered to be a native one if its root-mean-square deviation with respect to the true binding pose is less than $2 \AA$. In CASF-2007 benchmark, each ligand was given a total of 100 poses generated from docking software packages, namely, GOLD,${ }^{70,71}$ Surflex, ${ }^{72,73}$ FLexX ${ }^{74}$ and Ligand-Fit. ${ }^{75}$ In CASF-2013, 100 poses for each ligand were generated from three docking programs, namely, GOLD v5.1 (https://www.ccdc.cam.ac.uk), Surflex-Dock provided in SYBYL v8.1 (https://www.certara.com/), and MOE v2011 (https:// www.chemcomp.com/). Note that the RMSD values in CASF-2007 were referred to as the standard RMSD algorithm. ${ }^{63}$ However, a method may fail to report correct RMSDs on structures with a certain symmetry. Therefore, property-matched RMSD (RMSD ${ }^{\text {mm }}$ ) values were provided in CASF-2013. ${ }^{64,65}$ In both benchmarks, there can be more than one "native" pose for each given ligand in the given dataset. Then if a method can identify any one of these native poses, it will be regarded as successful for the ligand. Docking power is evaluated by the number of ligands whose "native" poses are correctly identified.

3.2.4 Screening power-This assessment validates a scoring function's ability to discriminate a target protein's true binders from decoy structures. There are a total of 65 different proteins in benchmark CASF 2013. Each receptor has at least 3 true binders. In fact, some of the 195 ligands in CASF 2013 dataset might bind to more than one protein. ${ }^{64}$ 
Indeed, by searching through the ChEMBL database, one can show that 12 proteins have more than 3 true binders. ${ }^{64}$ Fortunately, for each target protein, all of 195 ligands are labeled either as a true binder or as a decoy in the CASF dataset. Furthermore, for each target protein, the best true binder judged by the highest experimental binding affinity is specified in the CASF dataset.

There are two kinds of screening power measurements. The task of the first screening power measurement is to find out the enrichment factor (EF) in $x \%$ top-ranked candidates:

$$
\mathrm{EF}_{x \%}=\frac{\text { Number of true binders among } x \% \text { top-ranked candidates }}{\text { Total number of true binders of the given target protein }} .
$$

Here, top-ranked candidates are defined as those scoring function predicted candidates that have high binding affinities. The average of all $\mathrm{EF}$ values over 65 target proteins is used to assess the screening power of a scoring function.

The task of the second screening power measurement is to identify the best true binder. The success rate is given by the percentage of the best binders of 65 receptors being found among $x \%$ top-ranked candidates.

\section{Result and discussion}

Herein we assess the scoring power, ranking power, docking power and screening power of the proposed algebraic graph learning (AGL) approach using aforementioned benchmark datasets and evaluation metrics.

\subsection{Model parametrization}

For the sake of convenience, we use notation $\mathrm{AGL}_{\Omega, \beta, \tau}^{\mathscr{M}}$ to indicate the algebraic graph learning features generated by using interactive matrix type $\mathscr{M}$ with kernel type $\Omega$ and corresponding kernel parameters $\beta$ and $\tau$. As such, $\mathscr{M}=\operatorname{Adj}, \mathscr{M}=$ Lap , and $\mathscr{M}=\operatorname{Inv}$ represent adjacency matrix, Laplacian matrix, and pseudo inverse of Laplacian matrix, respectively. Here, $\Omega=\mathrm{E}$ and $\Omega=\mathrm{L}$ refer to generalized exponential and generalized Lorentz kernels, respectively. Additionally, $\beta$ is the kernel order such that $\beta=\kappa$ if $\Omega=\mathrm{E}$, and $\beta=\nu$ if $\Omega=\mathrm{L}$. Finally, $\tau$ is used such that $\eta_{k k^{\prime}}=\tau\left(\bar{r}_{k}+\bar{r}_{k^{\prime}}\right)$, where $\bar{r}_{k}$ and $\bar{r}_{k^{\prime}}$ are the van der Waals radii of element type $\mathrm{k}$ and element type $k^{\prime}$, respectively.

We propose an AGL representation in which multiple kernels are parametrized at different scale $(\eta)$ values. In this work, we consider at most two kernels. As a straightforward notation extension, two kernels can be parametrized by $\mathrm{AGL}_{\Omega_{1}, \beta_{1}, \tau_{1} ; \Omega_{2}, \beta_{2}, \tau_{2}}^{\mathscr{M}_{1} \mathscr{M}_{2}}$.

\subsection{Hyperparameter optimization}

As a rule of thumb, the machine learning models achieve the best performance when their essential parameters are properly optimized. To this end, 5-fold cross-validation (CV) is carried out to tune the kernel hyperparameters $\mathscr{M}, \Omega, \beta$, and $\tau$ in the proposed model 
$\mathrm{AGL}_{\Omega, \beta, \tau}^{\mathscr{M}}$. For simplicity, we perform the kernel parameter optimization on CASF-2007 benchmark's training data $(N=1105)$, the smallest training set among three benchmarks. Based on our previous work, ${ }^{42,45}$ a reasonable range of hyperparameters is listed in Table 2 . Specifically, the scale factor $\tau$ and power parameters $\beta=\kappa$ or $v$ are chosen in $[0.5,6]$ with an increment of 0.5 so that our model can effectively represent the interactions between protein and ligand in a complex. In addition, high values of the power order such as $\beta \in\{10,15,20\}$ are also taken into account to approximate the ideal low-pass filter (ILF). ${ }^{42}$ There are 40 element interactive pairs formed by the combinations of 4 commonly occurring atom types in proteins, i.e., $\{\mathrm{C}, \mathrm{N}, \mathrm{O}, \mathrm{S}\}$, and 10 commonly occurring atom types in ligands, i.e., $\{\mathrm{H}, \mathrm{C}$, $\mathrm{N}, \mathrm{O}, \mathrm{F}, \mathrm{P}, \mathrm{S}, \mathrm{Cl}, \mathrm{Br}, \mathrm{I}\}$. For adjacency matrices, we only consider their positive eigenvalues (Note that Laplacian matrices are positive semidefinite.). From the resulting set of eigenvalues or corresponding atomic descriptors, one can compute 9 descriptive statistical values, namely the sum, minimum (i.e., the Fiedler value for Laplacian matrices or the half band gap for adjacency matrices), maximum, mean, median, standard deviation, and variance of all eigenvalues. Additionally, we also utilize the number of eigenvalues and the sum of the second power of eigenvalues. This gives rise to a total of 360 features.

For a given interaction matrix type $\mathscr{M}$ and a given kernel type $\Omega$, we carry out 5 -fold cross validations on the training data of CASF-2007 to search for the optimal parameters $\beta$ and $\tau$ based on the averaged Pearson correlation coefficient value $\left(R_{p}\right)$. Figure $\mathrm{S} 1$ in the supplement material reports the best models with associated $R_{p}$ in this experiment. The optimal models are

$\left(\mathrm{AGL}_{\mathrm{E}, 6,2.5}^{\mathrm{Adj}}, R_{p}=0.748\right),\left(\mathrm{AGL}_{\mathrm{E}, 10,3.5}^{\mathrm{Lap}}, R_{p}=0.74\right),\left(\mathrm{AGL}_{\mathrm{E}, 1.5,4.5}^{\mathrm{Inv}}, R_{p}=0.708\right), \quad$ Among $\left(\mathrm{AGL}_{\mathrm{L}, 3.5,1.5}^{\mathrm{Adj}}, R_{p}=0.749\right),\left(\mathrm{AGL}_{\mathrm{L}, 15,3}^{\mathrm{Lap}}, R_{p}=0.740\right)$, and $\left(\mathrm{AGL}_{\mathrm{L}, 3.5,4}^{\mathrm{Inv}}, R_{p}=0.706\right)$.

them, $\mathrm{AGL}_{\mathrm{L}, 3.5,1.5}^{\mathrm{Adj}}$ is the best model and $\mathrm{AGL}_{\mathrm{L}, 3.5,4}^{\mathrm{Inv}}, R_{p}=0.706$ is the worst one. This finding is no surprise. In fact, adjacency matrix is the simplest one but still effectively captures all the interactions between protein and ligand atoms. Since the GNM-style matrix, i.e. $\mathscr{M}=$ Inv, involves the Moore-Penrose inverse, it likely admits errors from the numerical evaluation of large eigenvalues.

It is reported in the literature that the multiscale information can boost predictor's performance. ${ }^{40,45}$ Thus, on top of the optimal one-scale model, we impose another kernel with a different parametrization. We also carry out a similar grid-search procedure as we did for the single-scale model to explore optimal parameters. Based on Fig. S2 in the supplement material, the best two-kernel models are found at the following $\left(\mathrm{AGL}_{\mathrm{E}, 6,2.5 ; \mathrm{E}, 4,2}^{\mathrm{Adj}}, R_{p}=0.75\right),\left(\mathrm{AGL}_{\mathrm{E}, 10,3.5, \mathrm{E}, 5,1.5}^{\mathrm{Lap}}, R_{p}=0.745\right), \quad$ It is clear that models $\left(\mathrm{AGL}_{\mathrm{E}, 1.5,4.5 ; \mathrm{E}, 4.5,2}^{\mathrm{Inv}}, R_{p}=0.714\right),\left(\mathrm{AGL}_{\mathrm{L}, 3.5,1.5 ; \mathrm{L}, 15,0.5}^{\mathrm{Adj}}, R_{p}=0.751\right)$, $\left(\mathrm{AGL}_{\mathrm{L}, 15,3 ; \mathrm{L}, 6,1}^{\mathrm{Lap}}, R_{p}=0.745\right)$, and $\left(\mathrm{AGL}_{\mathrm{L}, 3.5,4 ; \mathrm{L}, 10,1}^{\mathrm{Inv}}, R_{p}=0.715\right)$. involving the adjacency matrix, i.e., $\mathrm{AGL}_{\mathrm{E}, 6,2.5 ; \mathrm{E}, 4,2}^{\mathrm{Adj}}$ and $\mathrm{AGL}_{\mathrm{L}, 3.5,1.5 ; \mathrm{L}, 15,0.5}^{\mathrm{Adj}}$, still outperform the rest. Finally, we form a consensus model named AGL-Score that is defined by the mean of the predicted values produced by those two aforementioned AGL models. 


\subsection{Performance and discussion}

4.3.1 Scoring power-First of all, we validate the scoring power of the proposed AGLScore using the CASF-2007 benchmark. We train two AGL models, namely $\mathrm{AGL}_{\mathrm{E}, 6,2.5 ; \mathrm{E}, 4,2}^{\mathrm{Adj}}$ and $\mathrm{AGL}_{\mathrm{L}, 3.5,1.5 ; \mathrm{L}, 15,0.5}^{\mathrm{Adj}}$ on the refined set $(N=1105)$ of the PDBbind v2007 excluding the test set $(N=195)$ of CASF-2007 benchmark. For the prediction task, we repeat each AGL model up to 50 times. The average of all the predicted values is used as the predicted binding affinity of the AGL model. It is noted that the energy unit in the PDBbind database is $\mathrm{pKd}$. For the $\mathrm{kcal} / \mathrm{mol}$ unit conversion, we multiply the predicted values by $-1.3633 .{ }^{57}$ In addition, we are interested in comparing the predictive power of our AGL-Score with various state-of- the-art scoring functions introduced in the literature. 36,53,58,63,76-78 Fig. 3a illustrates such a comparison. Clearly, the proposed model is one of the most accurate scoring functions in this benchmark with a Pearson correlation coefficient value $R_{p}=0.830$ and RMSE $=1.864 \mathrm{kcal} / \mathrm{mol}$. The runner-up is TNet-BP with reported $R_{p}$ $=0.826^{36}$. The geometric graph approach has a slightly lower performance with $R_{p}=$ $0.825^{45}$. This comparison confirms the scoring power of the present model. Furthermore, the correlation between our predicted values and the experimental data is depicted in Fig. 4a.

In the second benchmark, i.e. CASF-2013, its training data $(N=3516)$ is compiled based on the refined set of PDBbind v2015 excluding its test set $(N=195)$. We carry out a similar prediction procedure as of the previous one. Interestingly, the proposed model is able to outperform the state-of-the-art scoring functions adopted from Refs. ${ }^{58,64,78-80}$ as seen in Fig. 5b. Specifically, our AGL-Score attains Pearson correlation coefficient value $R_{p}=0.792$ and $\mathrm{RMSE}=1.973 \mathrm{kcal} / \mathrm{mol}$ followed by ElC-Score model with $R_{p}=0.774^{78}$. Our previous geometric graph model achieves similar performance to AGL-Score with $R_{p}=0.782^{45}$. In addition, Fig. $4 \mathrm{~b}$ provides a scatter plot to illustrate the correlation between our predicted values and experimental results.

CASF-2016 is the last benchmark considered in this work. It is also the latest CASF released by PDBbind database. ${ }^{66}$ We train the AGL model based on the refined set of PDBbind v2016 excluding the benchmark's test set. In this experiment, both training data $(N=3772)$ and test data $(N=285)$ are slightly larger than their predecessor, CASF-2013. The performance of a number of scoring functions has been reported for this benchmark. Specially, $\mathrm{K}_{\text {DEEP }},{ }^{81}$ Pafnucy, ${ }^{82}$ and PLEC-nn ${ }^{80}$ scoring functions make use of deep learning architectures. Also, numerous scoring function models have been tested by the PDBbind team. ${ }^{66}$ We compare the proposed AGL-Score to the aforementioned methods in Fig. 6. Note that, $\mathrm{K}_{\mathrm{DEEP}}$, Pafnucy, PLEC-nn, and EIC-Score all carry out the predictions on the original PDBbind v2016 core set with $N=290$. Table S1 in the Supporting Information provides the discrepancy information between the PDBbind v2016 core set and CASF-2016 test set. Our AGL-Score is still superior to its counterparts with $R_{p}=0.833(0.835)$ and RMSE $=1.733(1.732) \mathrm{kcal} / \mathrm{mol}$ for CASF-2016 test set (PDBbind v2016 core set). The second best approaches in the chart are $K_{\mathrm{DEEP}}$ and PLEC-nn both having $R_{p}=0.82$. The features and machine learning algorithms of these scoring functions are listed in Table 3. Moreover, the algebraic graph model's performance on this benchmark is still superior to that of the earlier geometric graph approach with $R_{p}=0.815^{45}$. This result confirms the accuracy and reliability of the AGL model for diversified binding affinity datasets. Finally, 
the comparison between the predicted affinities of AGL model and the experimental values is depicted in Fig. 4c.

4.3.2 Ranking power-We use predicted binding affinities on the scoring power task to validate the performance of our proposed scoring function on the ranking power. Among two models, namely generalized exponential kernel model AGL $\mathrm{Adj}, 6,2.5 ; \mathrm{E}, 4,2$ and generalized Lorentz kernel model AGL $\mathrm{Adj}_{\mathrm{L}, 3.5,1.5 ; \mathrm{L}, 15,0.5}$, the generalized exponential model produces better results on both benchmarks (see Figs. S3 and S4 in Supporting Information). Thus, its results are used in Fig. 3b and Fig. 5b. In CASF-2013, AGL-Score achieved the best performance with the high-level success measurement being 60\%, followed by X-ScoreHM with the success rate being 59\%. In CASF-2007, the AGL-Score's rate for the high-level success measurement is $54 \%$, which is ranked the third, following X-Score::HSScore (success rate $=58 \%)^{63}$ and $\Delta_{\text {vina }} R_{20}$ (success rate $=57 \%$ ). One can see that top rank scoring functions for the scoring power also perform quite well in the ranking power assessment. For example, in both CASF-2007 and CASF-2013, our AGL-Score model is among the top rank scoring functions on both scoring power and ranking power. If the machine learning models are excluded in the scoring tests, one can conclude that $\Delta_{\mathrm{vina}} \mathrm{RF}_{20}$ and $\mathrm{X}$-Score ${ }^{\mathrm{HM}}$ are good performers on these assessments.

4.3.3 Docking power-In order for our scoring function based machine learning method to be able to recognize the "native" pose among the computer-generated 100 poses, there is a need to create a training set which includes diverse conformers. To this end, for each given target ligand binding to a specific receptor, we use GOLD v5.6.3 ${ }^{71}$ to generate a set of 1000 training poses. The parameters of the GOLD software are chosen as the following: autoscale $=1.5$, early_termination $=0$, and gold_fitfunc_path $=$ plp. For each of CASF-2007 and CASF- 2013, we have created a total of 365,000 training poses for machine learning analysis. These pose structures and their scores reported by GOLD are available at https://weilab.math.msu.edu/AGL-Score.

For each target ligand, we retrain AGL-Score model on the target-specifically generated 1000 poses and use docking software's scores as their labels. To reduce the calculation time cost, we employ single exponential kernel AGL-Score $\mathrm{AGL}_{\mathrm{E}, 6,2.5}^{\mathrm{Adj}}$. Figs. $3 \mathrm{c}$ and $5 \mathrm{c}$ reveal that our AGL-Score is the top performer on the docking power test. Specifically, the AGLScore's success rate in identifying the best pose as the native pose is $84 \%$ on CASF-2007, followed by GOLD::ASP $(82 \%)^{63}$ and $\Delta$ vinaRF $_{20}(80 \%) .{ }^{58}$ In CASF-2013, our AGL-Score is still ranked at the first place with its success rate $=90 \%$, followed by $\Delta \mathrm{vinaRF}_{20}(87 \%)^{58}$ and Autodock Vina (85\%). ${ }^{58}$

Moreover, we are interested in examining the quality of the training data generated by GOLD using ChemPLP score (ChemPLP@GOLD) in term of the success rate of the best pose. Specifically, ChemPLP@GOLD scores $67 \%$ and $82 \%$ on the data sets generated for CASF-2007 and CASF-2013, respectively. By employing such datasets, our AGL-Score improves the success rates to $84 \%$ and $90 \%$ on CASF-2007 and CASF-2013, respectively. These statistical results strongly affirm that our AGL-Score is able to capture the real 
physical interactions in protein-ligand binding complexes and significantly improve the performance of existing docking software.

To do well on both scoring power and docking power tests is still a major challenge in the scoring function development. ${ }^{60,83,84}$ Most reputable docking software packages might offer reliable accuracy on pose predictions but perform poorly on binding affinity predictions. For example, for CASF-2007, GOLD software with ASP as a scoring function attains $82 \%$ in the success rate for the docking power task. However, it performs unsatisfactorily on the binding affinity prediction with Pearson correlation coefficient $\left(R_{p}\right)$ being 0.534 . On the other hand, RF-IChem, ${ }^{60}$ a SF based machine learning, achieves a more respectable $R_{p}=0.791$ on the scoring power test. However, it fails desperately on identifying the native pose task with the success rate being less than $30 \%$. Recently, another machine learning model named $\Delta$ vinaRF $F_{20}$ was developed. ${ }^{58}$ It produces a great performance on docking power task with the success rate being $80 \%$, while its Pearson correlation coefficient $\left(R_{p}=0.732\right)$ is also better than other traditional SFs. However, if one includes machine learning SFs, $\Delta v_{i n a R F} 20$ is still quite behind the best in the literature, i.e., TNet-BP $\left(R_{p}=0.826\right) .{ }^{36}$ Our AGL-Score not only achieves significant accuracy in the binding affinity prediction in various benchmarks ( $R_{p}=0.83$ in CASF-2007) but also attains the top place in the docking power assessment (success rate $=84 \%$ in CASF-2007). These results again rigorously confirm that the proposed eigenvalue features of multiscale weighted colored graphs have an ability to accurately encode the physical and biological information of protein-ligand complexes.

4.3.4 Screening power-In this assessment, our AGL-Score $\mathrm{AGL}_{\mathrm{E}, 6,2.5}^{\mathrm{Adj}}$ outperforms other scoring functions on both screening power measurements, namely enrichment factor (EF) and success rate at top $1 \%$ level on the CASF-2013 benchmark. Specifically, the AGLScore obtains the enrichment factor of 25.6. The methods at the second and third places are $\Delta$ vinaRF $_{20}(\mathrm{EF}=20.9)^{58}$ and GlideScore-SP $(\mathrm{EF}=19.5) .{ }^{64}$ On the success rate, our model is also the top performer with the success rate being $68 \% . \Delta v_{i n a R F} 20$ and GlideScore-SP are tied for the second place with a success rate being $60 \%$. Fig. 7 depicts the performance detail of various methods.

Since screening power assessment involves the identification of true binders for each of 65 proteins, we need to retrain our AGL-Score model. To this end, we collect a training set which includes both poses and energy labels to set up an AGL-Score model for each protein. Then, the poses of the 195 ligands are generated with a docking procedure and their energies are predicted by our AGL-Score model. The ligands with high energies are regarded as predicted binders.

Here, we describe our procedure for the training dataset selection and generation for the screening power test. The training set for each target protein consists of all complex structures and their energy labels from PDBbind v2015 refine set, excluding the core (test) set complexes. Additionally, for a given target protein, additional poses and their labels in the training set are generated by using Autodock Vina. ${ }^{85}$ For a given target protein, Autodock Vina is used to dock all the ligands in the PDBbind v2015 refined set, carefully excluding those in the core-set and true binders of the target protein. Autodock Vina is parametrized as follows: exhaustiveness $=10$, num_modes $=10$, and energy_range $=3$. Note 
that the list of true binders for each protein is listed in CASF 2013 benchmark. For each ligand, we keep the pose with the highest energy for the training set. This procedure gives rise to a few thousands of additional training poses and associated energy labels for each target protein.

Also, to use Autodock Vina score as the energy label, we convert kcal/mol to $\mathrm{pKd}$ unit by multiplying the Autodock Vina score with a conversion factor $-1.3633 .{ }^{57}$ Since those ligands in the refined set that do not bind to the given target protein are regarded as decoys, 64,65 their binding energies should not be larger than those of the true binders of the given target protein. Therefore, if the decoy energy predicted by Autodock Vina is higher than the lower bound of true binders' energies in the refined set database, we relabel the decoy with the lower bound of the true binders. The additional training set, i.e., Autodock Vina generated poses and their energy labels, can be downloaded via link https:// weilab.math.msu.edu/AGL-Score.

It is interesting to see that the performances of Autodock Vina are much lower than that of our AGL-Score. Specifically, EF of Autodock Vina's training set is 14.7 while that of AGLScore is 25.6. In addition, the success rate at top 1\% level of Autodock Vina's training set is $32 \%$ while AGL-Score gains more than $100 \%$ improvement with a success rate of $68 \%$. These results illustrate the remarkable improvement of our AGL-Score in the screening power in comparison to the well-known docking software.

The screening power assessment is still a difficult task for many machine learning based SF models. ${ }^{84}$ This type of SFs often performs well on scoring power test but works poorly on the identification of the true binders among the decoys. Particularly, the machine learning model RF@ML reported in ${ }^{84}$ achieves a reasonable $R_{p}=0.704$ on the scoring test for CASF-2013. This result is better than that of $\operatorname{AvinaRF}_{20}\left(R_{p}=0.686\right)$. However, RF@ML's performance on the screening task is not comparable to $\Delta \mathrm{vinaRF}_{20}$ as well as top docking scoring functions. Specifically, RF@ML attains EF at top 1\% level as low as 2.15, and success rate at top $1 \%$ level as low as 6.45 in CASF-2013. GlideScore-SP, by contrast, produces much higher EF (19.5) and success rate (60\%). By utilizing the superior algebraic graph representation features and appropriate training set, our AGL-Score is able to deliver top rankings in both screening power assessments without sacrificing the scoring accuracy.

\section{Conclusion}

Algebraic graph theories are commonly used in the study of molecular and biomolecular systems. However, most algebraic graph theory-based models are not as competitive as another predictive models on the same tasks. ${ }^{33-36}$ Even for some powerful methods on binding affinity predictions, they often perform poorly on other tasks involving unphysical and unreliable structures ${ }^{60}$. Motivated by our previous work on the multigraph approaches for B-factor predictions ${ }^{46}$ we propose a novel algebraic graph learning score (AGL-Score) for dealing with drug design related problems. The proposed AGL-Score model makes use of multiscale weight colored subgraphs to encode the essential physical and biological information, such as hydrogen bonds, electrostatics, van der Waals interactions, hydrophilicity, and hydrophobicity in the high-dimension space into the low-dimension 
representation of molecular and biomolecular structures. The constructions of three types of subgraphs are discussed in this work, namely adjacency matrix, Laplacian matrix, and pseudo-inverse of Laplacian matrix. The eigenvalues calculated from such matrices are used as features to characterize the biological and physical interactions of molecules and biomolecules.

In this work, we first investigate binding affinity datasets to demonstrate the robustness, accuracy, and reliability of the proposed model. To this end, three mainstream benchmark tests on scoring power, ranking power, docking power, and screening power assessments, namely CASF-2007, ${ }^{63}$ CASF-2013, ${ }^{64,65}$ and CASF-2016,, 66 are utilized. The results of benchmark tests reveal the superior performance of the proposed AGL-Score over other state-of-the-art methods. Additionally, we consider the ranking power, docking power and screening power assessments proposed in the literature. ${ }^{63,64}$ Additional training sets are generated to retrain the proposed AGL-Score for docking power and screening power assessments. Extensive numerical experiments rigorously confirm the top performance of the proposed AGL-Score model on benchmark protein-ligand binding datasets, containing both X-ray crystal and diverse decoy structures.

There has been widely spread scepticism or misunderstanding of the ability of machine learning-based scoring functions for docking and virtual screening. ${ }^{60}$ The present work shows that our machine learning method is not only able to enrich virtual screening but also able to significantly improve the performance of standard docking software packages, such as AutoDock Vina.

In addition to the confirmed accuracy and reliability, another major advantage of the present AGL-Score model is its simplicity. Only raw structural inputs regarding atom types and coordinates are used for free energy predictions. There is no need for any molecular force field. Moreover, the present AGL-Score model is robust without invoking complicated data pre-processing and optimization procedures.

\section{Supplementary Material}

Refer to Web version on PubMed Central for supplementary material.

\section{Acknowledgement}

This work was supported in part by NSF Grants DMS-1721024 and DMS-1761320, and NIH grant GM126189. DDN and GWW are also funded by Bristol-Myers Squibb and Pfizer.

\section{References}

(1). Hosoya H Topological Index. a Newly Proposed Quantity Characterizing the Topological Nature of Structural Isomers of Saturated Hydrocarbons. Bull. Chem. Soc. Jpn. 1971, 44, 2332-2339.

(2). Hansen PJ; Jurs PC Chemical Applications of Graph Theory. Part I. Fundamentals and Topological Indices. J. Chem. Educ. 1988, 65, 574.

(3). Bahar I; Atilgan AR; Erman B Direct Evaluation of Thermal Fluctuations in Proteins Using a Single-parameter Harmonic Potential. Folding Des. 1997, 2, 173-181.

(4). Yang LW; Chng CP Coarse-grained Models Reveal Functional Dynamics-I. Elastic Network Models-theories, Comparisons and Perspectives. Bioinf. Biol. Insights 2008, 2, 25-45. 
(5). Newman M Networks: An Introduction; Oxford university press: New York, NY, 2010.

(6). Bavelas A Communication Patterns in Task-Oriented Groups. J. Acoust. Soc. Am. 1950, 22, 725730.

(7). Dekker A Conceptual Distance in Social Network Analysis. J. Soc. Struct. 2005, 6.

(8). Wei GW; Zhan M; Lai CH Tailoring Wavelets for Chaos Control. Phys. Rev. Lett. 2002, 89, 284103.

(9). Kac M Can One Hear the Shape of a Drum? Am. Math. Mon. 1966, 73, 1-23.

(10). Trinajstic N Chemical Graph Theory; CRC Press: Boca Raton, FL, 1983

(11). Schultz HP Topological Organic Chemistry. 1. Graph Theory and Topological Indices of Alkanes. J. Chem. Inf. Comput. Sci. 1989, 29, 227-228.

(12). Janezic D; Milicevic A; Nikolic S; Trinajstic N Graph-Theoretical Matrices in Chemistry; CRC Press: Boca Raton, FL, 2015.

(13). Angeleska A; Jonoska N; Saito M DNa Rearrangement Through Assembly Graphs. Discrete. Appl. Math. 2009, 157, 3020-3037.

(14). Go N; Noguti T; Nishikawa T Dynamics of A Small Globular Protein in Terms of Low-frequency Vibrational Modes. Proc. Natl. Acad. Sci. 1983, 80, 3696-3700. [PubMed: 6574507]

(15). Tasumi M; Takenchi H; Ataka S; Dwidedi AM; Krimm S Normal Vibrations of Proteins: Glucagon. Biopolymers 1982, 21, 711-714. [PubMed: 7066480]

(16). Brooks BR; Bruccoleri RE; Olafson BD; States D; Swaminathan S; Karplus M CHARMM: A Program for Macromolecular Energy, Minimization, and Dynamics Calculations. J. Comput. Chem. 1983, 4, 187-217.

(17). Levitt M; Sander C; Stern PS Protein Normal-mode Dynamics: Trypsin Inhibitor, Crambin, Ribonuclease and Lysozyme. J. Mol. Biol. 1985, 181, 423-447. [PubMed: 2580101]

(18). Flory PJ Statistical Thermodynamics of Random Networks. Proc. Roy. Soc. Lond. A. 1976, 351, 351-378.

(19). Bahar I; Atilgan AR; Demirel MC; Erman B Vibrational Dynamics of Proteins: Significance of Slow and Fast Modes in Relation to Function and Stability. Phys. Rev. Lett. 1998, 80, $2733-$ 2736.

(20). Atilgan AR; Durrell SR; Jernigan RL; Demirel MC; Keskin O; Bahar I Anisotropy of Fluctuation Dynamics of Proteins with an Elastic Network Model. Bio-phys. J. 2001, 80, 505-515.

(21). Hinsen K Analysis of Domain Motions by Approximate Normal Mode Calculations. Proteins 1998, 33, 417-429. [PubMed: 9829700]

(22). Tama F; Sanejouand YH Conformational Change of Proteins Arising from Normal Mode Calculations. Protein Eng. 2001, 14, 1-6. [PubMed: 11287673]

(23). Cui Q; Bahar I Normal Mode Analysis: Theory and Applications to Biological and Chemical Systems; Chapman and Hall/CRC: Boca Raton, FL, 2010.

(24). Balaban AT Chemical Applications of Graph Theory; Academic Press: Cambridge, MA, 1976.

(25). Foulds LR Graph Theory Applications; Springer Science \& Business Media: New York, NY, 2012.

(26). Ozkanlar A; Clark AE ChemNetworks: A Complex Network Analysis Tool for Chemical Systems. J. Comput. Chem. 2014, 35, 495-505. [PubMed: 24311311]

(27). Di Paola L; Giuliani A Protein Contact Network Topology: A Natural Language for Allostery. Curr. Opin. Struct. Biol. 2015, 31, 43-48. [PubMed: 25796032]

(28). Canutescu AA; Shelenkov AA; Dunbrack RL A Graph-Theory Algorithm for Rapid Protein Side-Chain Prediction. Protein Sci. 2003, 12, 2001-2014. [PubMed: 12930999]

(29). Ryslik GA; Cheng Y; Cheung K-H; Modis Y; Zhao H A Graph Theoretic Approach to Utilizing Protein Structure to Identify Non-Random Somatic Mutations. BMC Bioinf. 2014, 15, 86.

(30). Jacobs DJ; Rader AJ; Kuhn LA; Thorpe MF Protein Flexibility Predictions Using Graph Theory. Proteins 2001, 44, 150-165. [PubMed: 11391777]

(31). Vishveshwara S; Brinda K; Kannan N Protein Structure: Insights from Graph Theory. J. Theor. Comput. Chem. 2002, 1, 187-211. 
(32). Wu Z; Ramsundar B; Feinberg EN; Gomes J; Geniesse C; Pappu AS; Leswing K; Pande V MoleculeNet: A Benchmark for Molecular Machine Learning. arXiv preprint arXiv:1703.00564 2017

(33). Pires DEV; Ascher DB; Blundell TL MCSM: Predicting the Effects of Mutations in Proteins Using Graph-Based Signatures. Struct. Bioinf. 2014, 30, 335-342.

(34). Quan L; Lv Q; Zhang Y STRUM: Structure-Based Prediction of Protein Stability Changes upon Single-Point Mutation. Bioinformatics 2016, 32, 2936-2946. [PubMed: 27318206]

(35). Cang ZX; Wei GW Analysis and Prediction of Protein Folding Energy Changes upon Mutation by Element Specific Persistent Homology. Bioinformatics 2017, 33, 3549-3557. [PubMed: 29036440]

(36). Cang ZX; Wei GW TopologyNet: Topology Based Deep Convolutional and Multitask Neural Networks for Biomolecular Property Predictions. PLoS Comput. Biol. 2017, 13(7), e1005690, 10.1371/journal.pcbi.1005690.

(37). Park JK; Jernigan R; Wu Z Coarse Grained Normal Mode Analysis Vs. Refined Gaussian Network Model for Protein Residue-Level Structural Fluctuations. Bull. Math. Biol. 2013, 75, 124-160. [PubMed: 23296997]

(38). Xia K; Wei G-W A Review of Geometric, Topological and Graph Theory Apparatuses for the Modeling and Analysis of Biomolecular Data. arXiv preprint arXiv:1612.01735 2016 ,

(39). Opron K; Xia KL; Wei GW Fast and Anisotropic Flexibility-Rigidity Index for Protein Flexibility and Fluctuation Analysis. J. Chem. Phys. 2014, 140, 234105.

(40). Opron K; Xia KL; Wei GW Communication: Capturing Protein Multiscale Thermal Fluctuations. J. Chem. Phys. 2015, 142.

(41). Nguyen DD; Xia KL; Wei GW Generalized Flexibility-Rigidity Index. J. Chem. Phys. 2016, 144, 234106.

(42). Xia KL; Opron K; Wei GW Multiscale Gaussian Network Model (mGNM) and Multiscale Anisotropic Network Model (mANM). J. Chem. Phys. 2015, 143, 204106.

(43). Edelsbrunner H; Letscher D; Zomorodian A Topological Persistence and Simplification. Discrete Comput. Geom. 2002, 28, 511-533.

(44). Zomorodian A; Carlsson G Computing Persistent Homology. Discrete Comput. Geom. 2005, 33, 249-274.

(45). Nguyen DD; Xiao T; Wang ML; Wei GW Rigidity strengthening: A mechanism for proteinligand binding. J. Chem. Inf. Model. 2017, 57, 1715-1721. [PubMed: 28665130]

(46). Bramer D; Wei GW Weighted Multiscale Colored Graphs for Protein Flexibility and Rigidity Analysis. J. Chem. Phys. 2018, 148, 054103.

(47). Zheng Z; Merz KM Jr Ligand Identification Scoring Algorithm (LISA). J. Chem. Inf. Model. 2011, 51, 1296-1306. [PubMed: 21561101]

(48). Verkhivker G; Appelt K; Freer ST; Villafranca JE Empirical Free Energy Calculations of LigandProtein Crystallographic Complexes. I. Knowledge Based Ligand Protein Interaction Potentials Applied to the Prediction of Human Immunodeficiency Virus Protease Binding Affinity. Protein Eng. 1995, 8, 677-691. [PubMed: 8577696]

(49). Eldridge MD; Murray CW; Auton TR; Paolini GV; Mee RP Empirical Scoring Functions: I. the Development of a Fast Empirical Scoring Function to Estimate the Binding Affinity of Ligands in Receptor Complexes. J. Comput. Aided. Mol. Des. 1997, 11, 425-445. [PubMed: 9385547]

(50). Muegge I; Martin Y A General and Fast Scoring Function for Protein-Ligand Interactions: A Simplified Potential Approach. J. Med. Chem. 1999, 42, 791-804. [PubMed: 10072678]

(51). Velec H; Gohlke H; Klebe G DrugScore (CSD)-Knowledge-Based Scoring Function Derived from Small Molecule Crystal Data with Superior Recognition Rate of Near-Native Ligand Poses and Better Affinity Prediction. J. Med. Chem. 2005, 48, 6296-303. [PubMed: 16190756]

(52). Huang SY; Zou X An Iterative Knowledge-Based Scoring Function to Predict Protein-Ligand Interactions: I. Derivation of Interaction Potentials. J. Comput. Chem. 2006, 27, 1865-1875.

(53). Ballester PJ; Mitchell JBO A Machine Learning Approach to Predicting Protein - Ligand Binding Affinity with Applications to Molecular Docking. Bioinformatics 2010, 26, 1169-1175. [PubMed: 20236947] 
(54). Kinnings SL; Liu N; Tonge PJ; Jackson RM; Xie L; Bourne PE A Machine Learning Based Method to Improve Docking Scoring Functions and Its Application to Drug Repurposing. J. Chem. Inf. Model. 2011, 51, 408-419. [PubMed: 21291174]

(55). Ashtawy HM; Mahapatra NR A Comparative Assessment of Ranking Accuracies of Conventional and Machine-Learning-Based Scoring Functions for Protein-Ligand Binding Affinity Prediction. IEEE/ACM Trans. Comput. Biol. Bioinf. 2012, 9, 1301-1313.

(56). Lee AA; Brenner MP; Colwell LJ Predicting Protein-Ligand Affinity with a Random Matrix Framework. Proc. Natl. Acad. Sci. 2016, 201611138.

(57). Wang B; Zhao Z; Nguyen DD; Wei GW Feature Functional Theory - Binding Predictor (FFT-BP) for the Blind Prediction of Binding Free Energy. Theor. Chem. Acc. 2017, 136, 55.

(58). Wang C; Zhang Y Improving Scoring-Docking-Screening Powers of Protein-Ligand Scoring Functions Using Random Forest. J. Comput. Chem. 2017, 38, 169-177. [PubMed: 27859414]

(59). Nguyen DD; Cang Z; Wu K; Wang M; Cao Y; Wei G-W Mathematical Deep Learning for Pose and Binding Affinity Prediction and Ranking in D3R Grand Challenges. J. Comput.-Aided Mol. Des. 2019, 33, 71-82. [PubMed: 30116918]

(60). Gabel J; Desaphy J; Rognan D Beware of Machine Learning-Based Scoring Functions: On the Danger of Developing Black Boxes. J. Chem. Inf. Model. 2014, 54, 2807-2815. [PubMed: 25207678]

(61). Li Y; Yang J Structural and Sequence Similarity Makes a Significant Impact on MachineLearning-Based Scoring Functions for Protein-Ligand Interactions. J. Chem. Inf. Model. 2017, 57, 1007-1012. [PubMed: 28358210]

(62). Kramer C; Gedeck P Leave-Cluster-out Cross-Validation Is Appropriate for Scoring Functions Derived from Diverse Protein Data Sets. J. Chem. Inf. Model. 2010, 50, 1961-1969. [PubMed: 20936880]

(63). Cheng T; Li X; Li Y; Liu Z; Wang R Comparative Assessment of Scoring Functions on a Diverse Test Set. J. Chem. Inf. Model. 2009, 49, 1079-1093. [PubMed: 19358517]

(64). Li Y; Han L; Liu Z; Wang R Comparative Assessment of Scoring Functions on an Updated Benchmark: 2. Evaluation Methods and General Results. J. Chem. Inf. Model. 2014, 54, $1717-$ 1736. [PubMed: 24708446]

(65). Li Y; Su M; Liu Z; Li J; Liu J; Han L; Wang R Assessing Protein-Ligand Interaction Scoring Functions with the CASF-2013 Benchmark. Nat. Protoc. 2018, 13, 666. [PubMed: 29517771]

(66). Su M; Yang Q; Du Y; Feng G; Liu Z; Li Y; Wang R Comparative Assessment of Scoring Functions: The CASF-2016 Update. J. Chem. Inf. Model. 2018,

(67). Cang ZX; Mu L; Wei GW Representability of Algebraic Topology for Biomolecules in Machine Learning Based Scoring and Virtual Screening. PLoS Comput. Biol. 2018, 14(1), e1005929, 10.1371/journal.pcbi.1005929.

(68). Cang ZX; Wei GW Integration of Element Specific Persistent Homology and Machine Learning for Protein-ligand Binding Affinity Prediction. Int. J. Numer. Method. Biomed. Eng. 2018, 34(2), DOI: $10.1002 / \mathrm{cnm} .2914$.

(69). Liu J; Wang R Classification of Current Scoring Functions. J. Chem. Inf. Model. 2015, 55, 475482. [PubMed: 25647463]

(70). Jones G; Willett P; Glen RC Molecular Recognition of Receptor Sites Using a Genetic Algorithm with a Description of Desolvation. J. Mol. Biol. 1995, 245, 43-53. [PubMed: 7823319]

(71). Jones G; Willett P; Glen RC; Leach AR; Taylor R Development and Validation of a Genetic Algorithm for Flexible Docking. J. Mol. Biol. 1997, 267, 727-748. [PubMed: 9126849]

(72). Jain AN Surflex: Fully Automatic Flexible Molecular Docking Using a Molecular SimilarityBased Search Engine. J. Med. Chem. 2003, 46, 499-511. [PubMed: 12570372]

(73). Jain AN Surflex-Dock 2.1: Robust Performance from Ligand Energetic Modeling, Ring Flexibility, and Knowledge-Based Search. J. Comput.-Aided Mol. Des. 2007, 21, 281-306. [PubMed: 17387436]

(74). Rarey M; Kramer B; Lengauer T; Klebe G A Fast Flexible Docking Method Using an Incremental Construction Algorithm. J. Mol. Biol. 1996, 261, 470-489. [PubMed: 8780787] 
(75). Venkatachalam CM; Jiang X; Oldfield T; Waldman M LigandFit: A Novel Method for the ShapeDirected Rapid Docking of Ligands to Protein Active Sites. J. Mol. Graphics Modell. 2003, 21, 289-307.

(76). Li G-B; Yang L-L; Wang W-J; Li L-L; Yang S-Y ID-Score: A New Empirical Scoring Function Based on a Comprehensive Set of Descriptors Related to Protein-Ligand Interactions. J. Chem. Inf. Model. 2013, 53, 592-600. [PubMed: 23394072]

(77). Li H; Leung K-S; Wong M-H; Ballester PJ Improving AutoDock Vina Using Random Forest: The Growing Accuracy of Binding Affinity Prediction by the Effective Exploitation of Larger Data Sets. Mol. Inf. 2015, 34, 115-126.

(78). Nguyen DD; Wei G-W DG-GL: Differential Geometry-Based Geometric Learning of Molecular Datasets. Int. J. Numer. Method. Biomed. Eng. 2019, 35, e3179. [PubMed: 30693661]

(79). Li H; Leung K-S; Wong M-H; Ballester PJ Low-Quality Structural and Interaction Data Improves Binding Affinity Prediction via Random Forest. Molecules 2015, 20, 10947-10962. [PubMed: 26076113]

(80). Wójcikowski M; Kukielka M; Stepniewska-Dziubinska M; Siedlecki P Development of a Protein-Ligand Extended Connectivity (PLEC) Fingerprint and Its Application for Binding Affinity Predictions. Bioinformatics 2018

(81). Jiménez J; Skalic M; Martínez-Rosell G; De Fabritiis G K DEEP: Protein-Ligand Absolute Binding Affinity Prediction Via 3D-Convolutional Neural Networks. J. Chem. Inf. Model. 2018, 58, 287-296. [PubMed: 29309725]

(82). Stepniewska-Dziubinska MM; Zielenkiewicz P; Siedlecki P Development and Evaluation of a Deep Learning Model for Protein-Ligand Binding Affinity Prediction. Bioinformatics 2018, 1, 9.

(83). Plewczynski D; Lazniewski M; Augustyniak R; Ginalski K Can We Trust Docking Results? Evaluation of Seven Commonly Used Programs on PDBbind Database. J. Comput. Chem. 2011, 32, 742-755. [PubMed: 20812323]

(84). Khamis MA; Gomaa W Comparative Assessment of Machine-Learning Scoring Functions on PDBbind 2013. Eng. Appl. Artif. Intell. 2015, 45, 136-151.

(85). Trott O; Olson AJ AutoDock Vina: Improving the Speed and Accuracy of Docking with a New Scoring Function, Efficient Optimization, and Multithreading. J. Computat. Chem. 2010, 31, 455-461. 


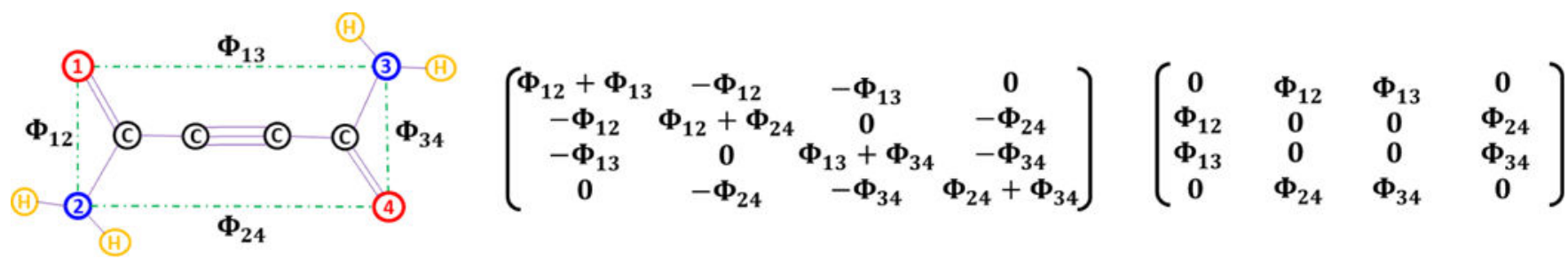

Figure 1:

Illustration of weighted colored subgraph $G_{\mathrm{NO}}(\mathrm{Left})$, its Laplacian matrix (Middle), and adjacency matrix (Right) for cellocidin molecule $\left(\mathrm{C}_{4} \mathrm{H}_{4} \mathrm{~N}_{2} \mathrm{O}_{2}\right)$. Graph vertices, namely oxygen (i.e., atoms 1 and 4) and nitrogen (i.e., atoms 2 and 3), are labeled in red and blue colors, respectively. Here, graph edges (i.e., $\Phi_{i j}$ ) are labeled by green-dashed lines which are not covalent bonds. Here, $\Phi_{i j}$ are distance-weighted edges. Note that there are 9 other nontrivial subgraphs for this molecule (i.e., $G_{\mathrm{CC}}, G_{\mathrm{CN}}, G_{\mathrm{CO}}, G_{\mathrm{GH}}, G_{\mathrm{NN}}, \mathrm{G}_{\mathrm{NH}}, \mathrm{G}_{\mathrm{OO}}, G_{\mathrm{OH}}$, $\left.G_{\mathrm{HH}}\right)$. 


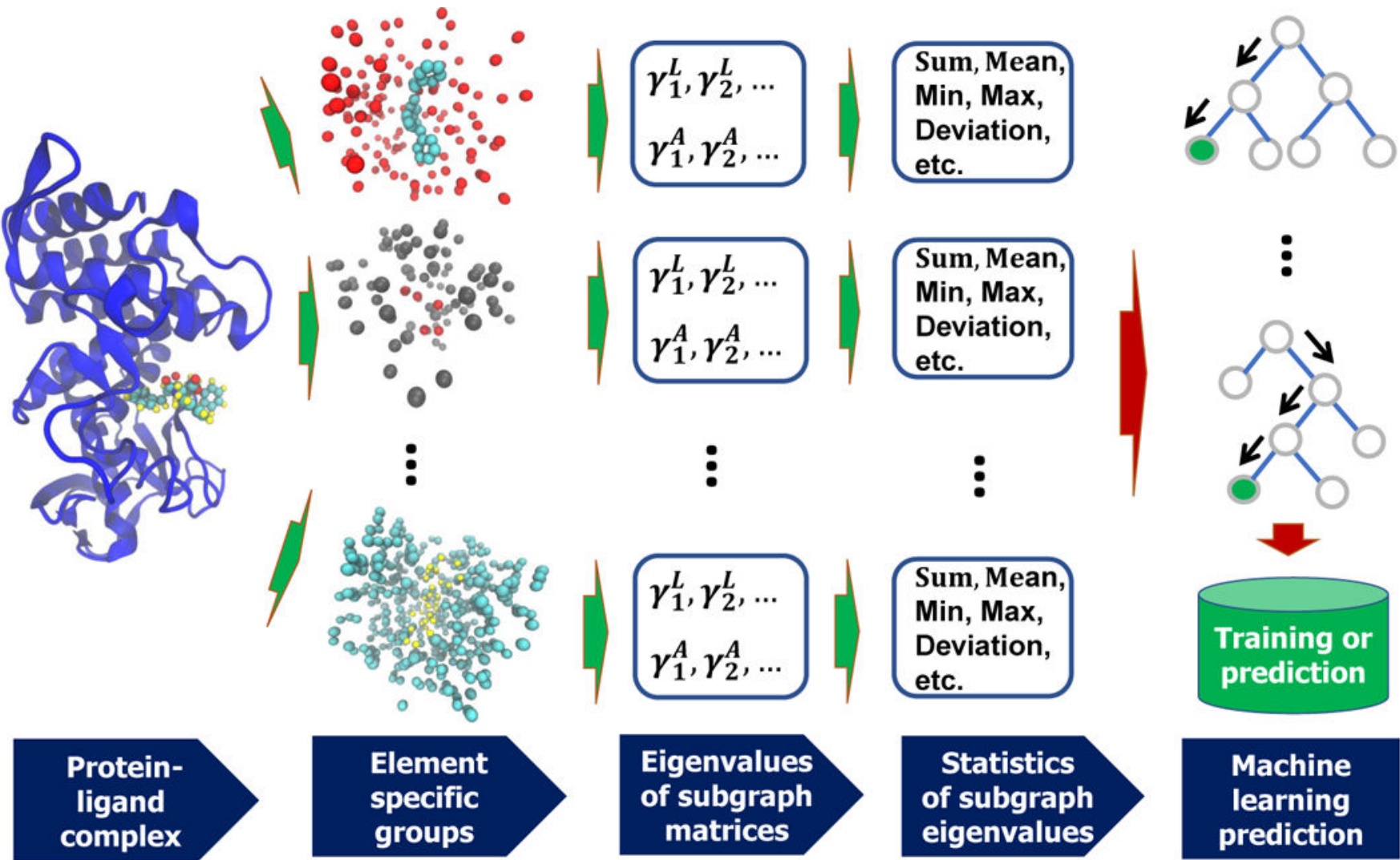

Figure 2:

Illustration of algebraic graph learning strategy using $1 \mathrm{OS} 0$ (first column). In the second column, element specific groups are, from top to bottom, $\mathrm{OC}, \mathrm{NO}$, and $\mathrm{CH}$, respectively. Their corresponding weighted labeled graph Laplacian and adjacency eigenvalues are shown in the third column. The statistics of these eigenvalues (fourth column) are used in gradient boosting trees for training and prediction (last column). 
a) Scoring Power (CASF-2007) TNet-BP. -0.826 EIC-Score RF::VinaElem ID-Score $\Delta_{\text {vina }} R_{20}$ SVR-Score X-Score::HMScore DrugScoreCSD SYBYL::ChemScore AutoDock Vina DS::PLP1 GOLD::ASP SYBYL::G-Score DS::LUDI3 DS::LigScore2 GlideScore-XP DS::PMF

GOLD::ChemScore SYBYL::D-Score DS::Jain

GOLD::GoldScore

0.295

SYBYL::PMF-Score- 0.268

SYBYL::F-Score- 0.216 0.817 0.753 0.732 0.726 0.644 0.569 0.555

0.554

0.545 0.534 0.492 0.487 0.464 0.457 0.445 0.441 0.392 .316 (1) b) X-Score:
Ranking Power (CASF-2007) ::HSScore $\Delta_{\text {vina }} R_{20}$ AGL-Score-

$\begin{array}{llll}0.4 & 0.6 & 0.8 & 1.0\end{array}$

Autodock Vina DS::PLP2

DrugScoreCSD SYBY SYBYL: SYBYL:

GOLD:ASP-

DS::LUDI3.

DS::Jain-

DS::PMF

SYBYL::PMF-Score-

GOLD::ChemScore-

DS::LigScore2

GlideScore-XP

SYBYL::F-Score-

GOLD::GoldScore- $23 \%$

$$
\begin{array}{llll}
30 & 40 & 50 & 60 \\
30
\end{array}
$$

c)

Docking Power (CASF-2007)

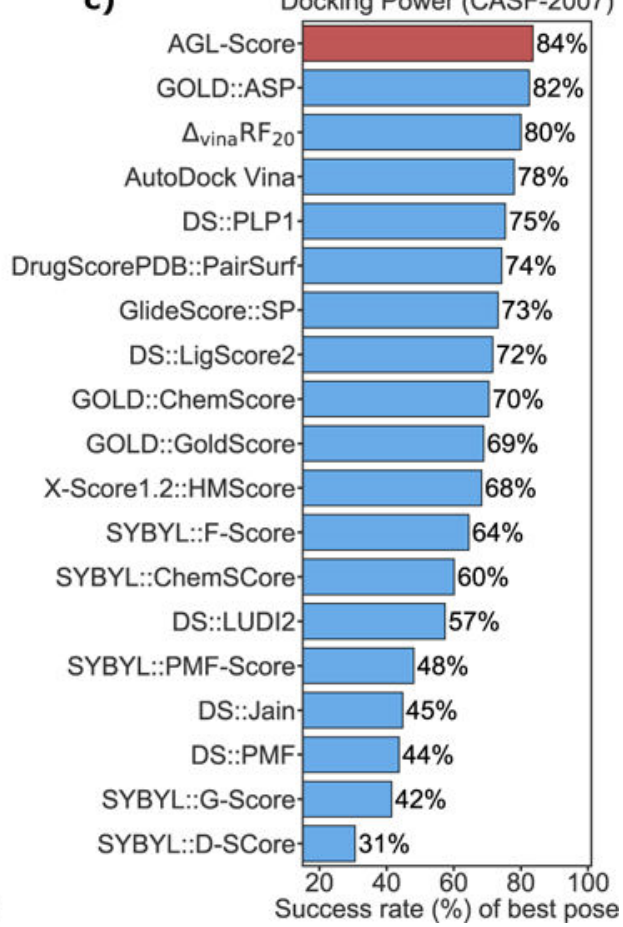

Figure 3:

Performance comparison of different scoring functions on CASF-2007 benchmark. a) scoring power measured by Pearson correlation coefficient; b) ranking power evaluated by the high-level success measurement, and c) docking power in term of the rate of successfully identifying the "native" pose from 100 poses for each ligand. The proposed algebraic graph learning based scoring function, AGL-Score, is plotted in the red color. The results of other methods, taken from Refs., ${ }^{36,53,58,63,76-78}$ are in the blue color. 
a)

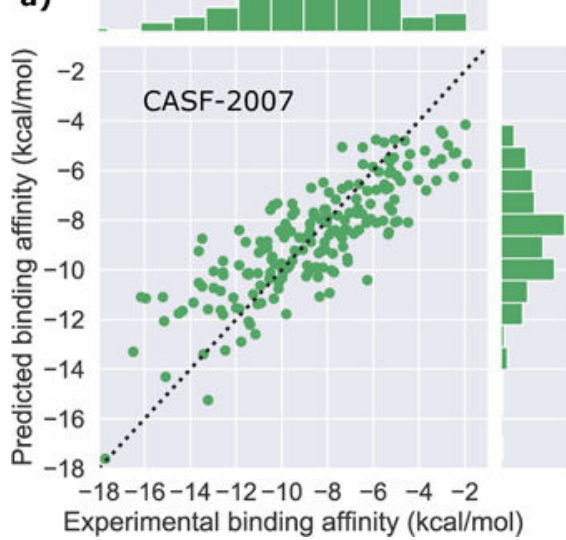

b)

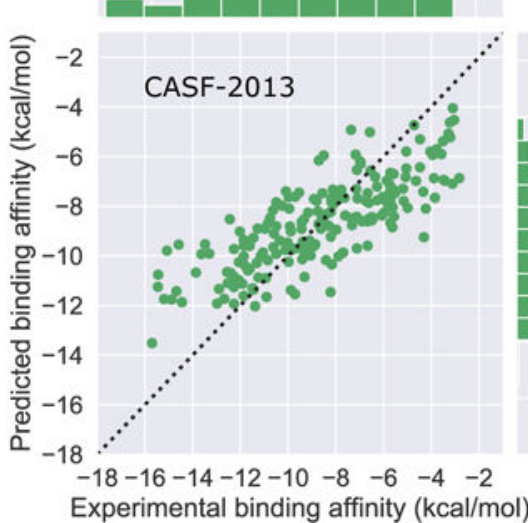

c)
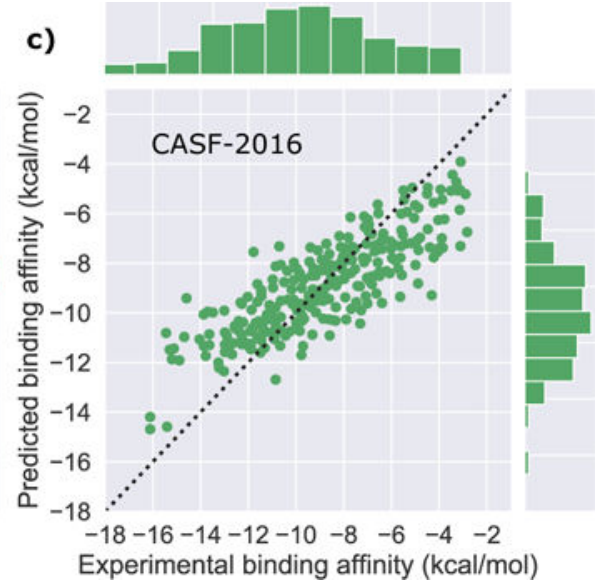

Figure 4:

Correlation between AGL-Score predictions and experimental data for various benchmarks.

a) CASF-2007: Pearson correlation coefficient $R_{p}=0.83$ and RMSE $=1.864 \mathrm{kcal} / \mathrm{mol}$; b)

CASF-2013: Pearson correlation coefficient $R_{p}=0.792$ and RMSE $=1.973 \mathrm{kcal} / \mathrm{mol}$; c)

CASF-2016: Pearson correlation coefficient $R_{p}=0.833$ and RMSE $=1.733 \mathrm{kcal} / \mathrm{mol}$. 
a) Scoring Power (CASF-2013) AGL-Score EIC-Score PLEC-nn RF::VinaElem $\Delta_{\text {vina }} \mathrm{RF}_{20}$ $\Delta_{\text {vina }} R_{2} F_{20}$
X-Score $\triangle \mathrm{SAS}$ ChemScore@SYBYL
ChemPLP@GOLD PLP1@DS

AutoDock Vina G-Score@SYBYL ASP@GOLD ASE@MOE

Chemscore@GOLD D-Score@SYBYL LUDI3@DS

GoldScore@GOLD Affinity-dG@MOE NHA LigScore2@DS GlideScore-SP Jain@DS PMF@DS

GlideScore-X ondon-dG@MOE-]0.242

PMF@SYBYL- 0.221

\section{b)}

\section{b)} AGL-Score

ChemPLP@GOLD 0.770
0.752
0.686 0.614
0.606
0.592 0.579 0.568
0.564 0.564
0.558 0.558
0.556 0.556
0.544 0.536 0.526 0.511 0.487 0.483
0.482 0.482 0.478
0.473
0.456 0.456 0.408 0.364 277 0.4 $\begin{array}{lll}0.4 & 0.6 & 0.8 \\ \text { Pearson's R }\end{array}$ $\Delta_{\text {vina }} R_{20}$ PLP2@DS

GoldScore@GOLD ChemScore@SYBYL Affinity-dG@MOE LigScore1@DS Alpha-HB@MOE G-Score@SYBYL LUDI1@DS AutoDock Vina D-Score@SYBYL $\triangle \mathrm{SAS}$ PMF@DS ASP@GOLD ChemScore@GOLD London-dG@MOE. PMF@SYBYL

GlideScore-SP Jain@DS. ASE@MOE GlideScore-XP.
Ranking Power (CASF-2013)

$$
3035404550556065
$$
Success rate (\%) at high-level c) Docking Power (CASF-2013)

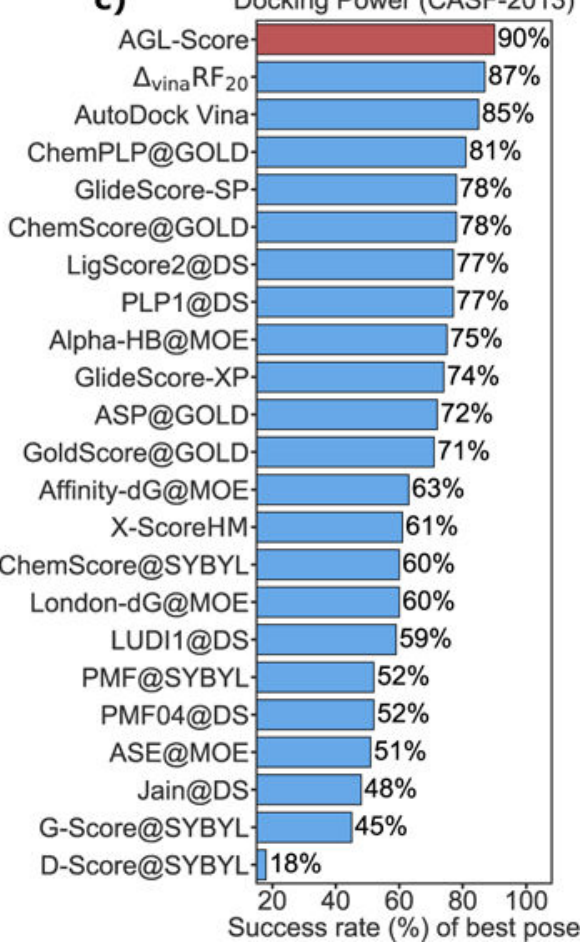

Figure 5:

Performance comparison of different scoring functions on CASF-2013 benchmark, a) the scoring power measured by Pearson correlation coefficient; $b$ ) the ranking power evaluated by the high-level success measurement, and c) the docking power in term of the rate of successfully identifying the "native" pose from 100 poses for each ligand. The proposed algebraic graph learning based scoring function, AGL-Score, is plotted in the red color. The results of other methods, taken from Refs., ${ }^{58,64,78-80}$ are in the blue color. 
Scoring Power (CASF-2016)

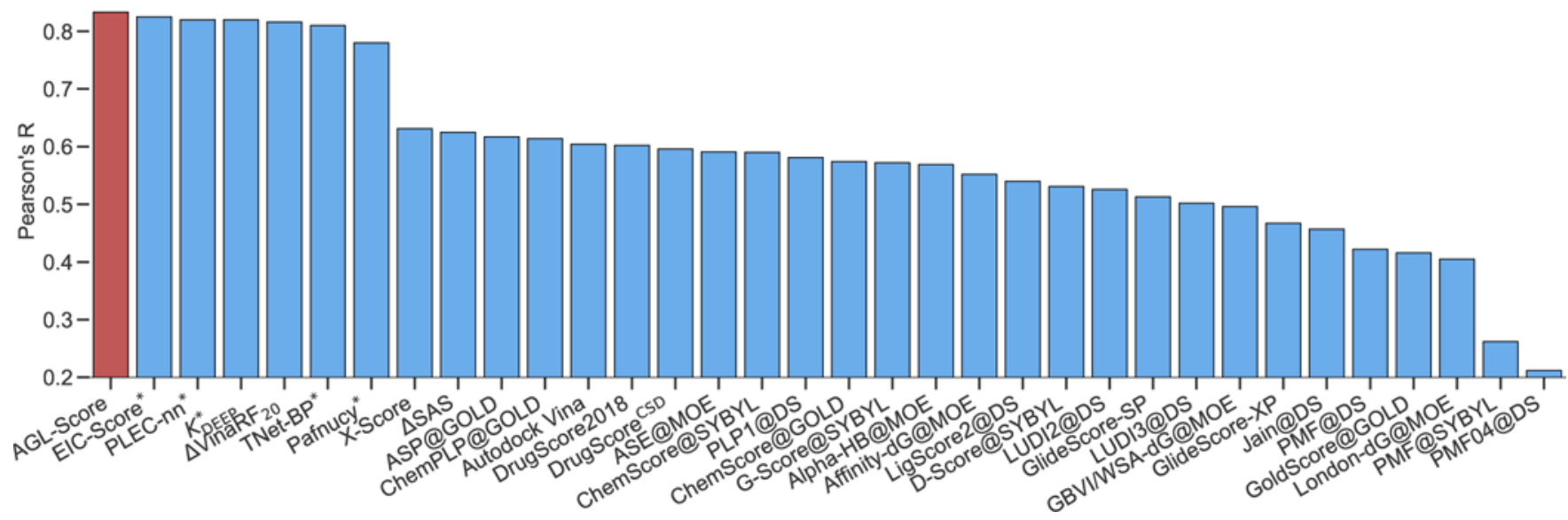

Figure 6:

Performance comparison of different scoring functions on CASF-2016. The Pearson correlation coefficients of other methods are taken from Refs. $36,66,78,80-82$ The proposed algebraic graph learning based scoring function, AGL-Score, achieves $R_{p}=0.833$ and $\mathrm{RMSE}=1.733 \mathrm{kcal} / \mathrm{mol}$. Note that, scoring functions marked with $*$ use PDBbind v2016 core set $(N=290)$. 
a)

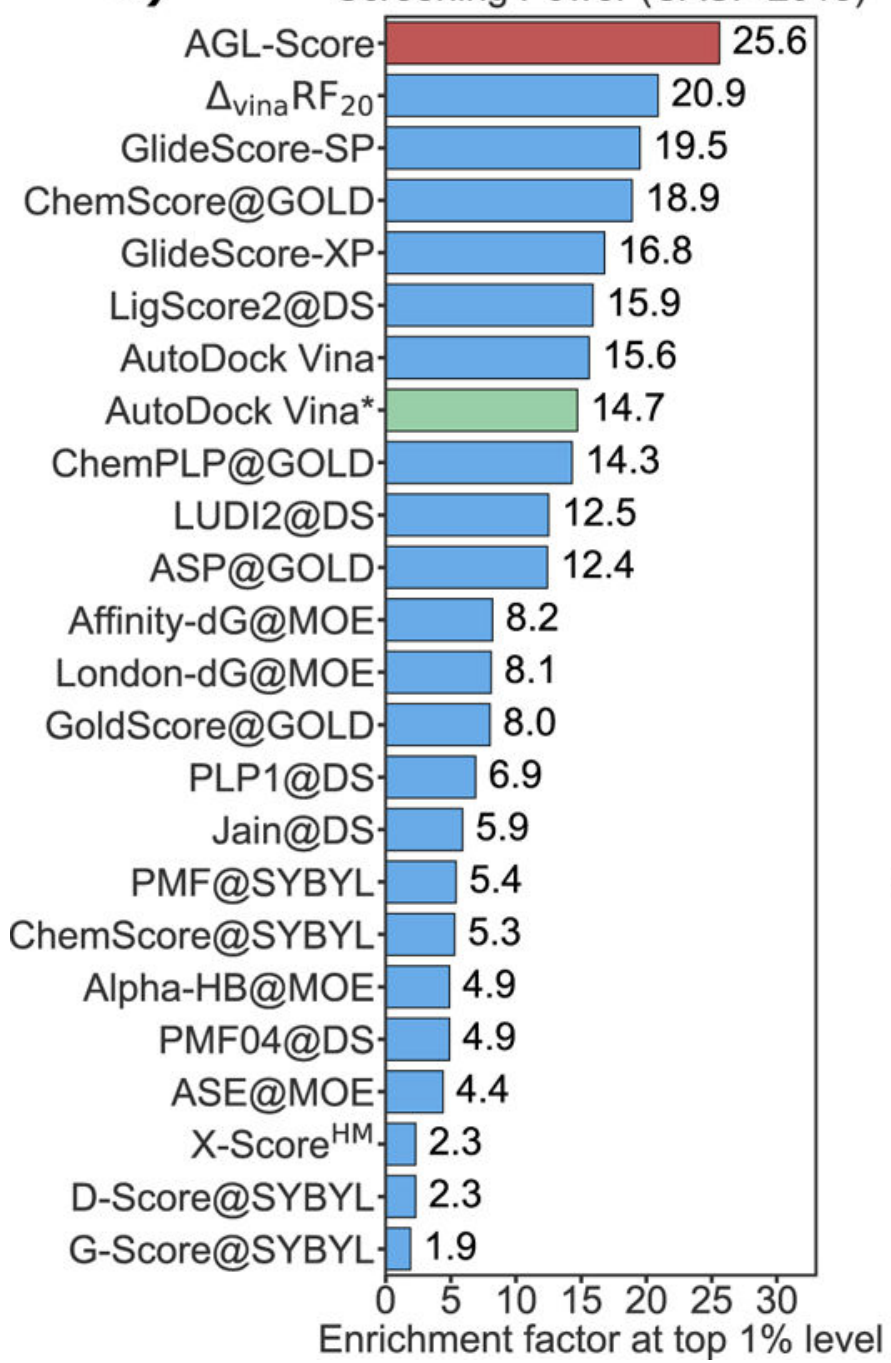

b)

Screening Power (CASF-2013)

\begin{tabular}{|c|c|}
\hline AGL-Score- & $68 \%$ \\
\hline$\Delta_{\text {vina }} R F_{20}$ & $60 \%$ \\
\hline GlideScore-SP. & $\square 60 \%$ \\
\hline GlideScore-XP. & $52 \%$ \\
\hline ChemScore@GOLD. & $49 \%$ \\
\hline LigScore2@DS & $48 \%$ \\
\hline AutoDock Vina- & $45 \%$ \\
\hline ChemPLP@GOLD - & $42 \%$ \\
\hline LUDI2@DS & $38 \%$ \\
\hline ASP@GOLD & $37 \%$ \\
\hline AutoDock Vina* & $\square 32 \%$ \\
\hline Affinity-dG@MOE- & $23 \%$ \\
\hline PLP1@DS & $22 \%$ \\
\hline GoldScore@GOLD & $22 \%$ \\
\hline London-dG@MOE. & $22 \%$ \\
\hline Jain@Ds & $17 \%$ \\
\hline ChemScore@SYBYL- & $15 \%$ \\
\hline Alpha-HB@MOE- & $14 \%$ \\
\hline PMF@SYBYL. & $14 \%$ \\
\hline PMF04@DS. & $12 \%$ \\
\hline ASE@MOE. & $12 \%$ \\
\hline X-Score ${ }^{H M}$ & $9 \%$ \\
\hline D-Score@SYBYL- & $6 \%$ \\
\hline G-Score@SYBYL. & $5 \%$ \\
\hline
\end{tabular}

Figure 7:

Performance comparison of different scoring functions on the screening power for CASF-2013 benchmark evaluated by a) enrichment factor and (b) success rate at the top $1 \%$ level. The proposed algebraic graph learning based scoring function, AGL-Score, is in the red color. The results of Autodock Vina computed in the present work (Autodock Vina*) is in the green color. The results of other methods, taken from Refs., ${ }^{58,64}$ are in the blue color. 
Table 1:

Summary of PDBbind datasets used in the present work

\begin{tabular}{|l|c|c|}
\hline & Training set complexes & Test set complexes \\
\hline CASF-2007 benchmark & 1105 & 195 \\
\hline CASF-2013 benchmark & 3516 & 195 \\
\hline CASF-2016 benchmark & 3772 & 285 \\
\hline
\end{tabular}


Table 2:

The ranges of model hyperparameters for 5-fold cross-validations

\begin{tabular}{|c|c|}
\hline Parameter & Domain \\
\hline$\tau$ & $\{0.5,1.0, \ldots, 6\}$ \\
\hline$\beta$ & $\{0.5,1.0, \ldots, 6\} \cup\{10,15,20\}$ \\
\hline $\mathscr{M}$ & $\{$ Adj, Lap, Inv $\}$ \\
\hline
\end{tabular}


Table 3:

Description of some machine learning-based scoring functions.

\begin{tabular}{|c|c|c|}
\hline Name & Features & Machine learning algorithms \\
\hline TNet-BP $^{36}$ & Algebraic topology & Convolution neural network (CNN) \\
EIC-Score $^{78}$ & Differential geometry & GBT \\
RI-Score & Random Forest \\
AGL-Score & Geometric graph & GBT \\
PLEC-nn & Algebraic graph & Neural networks \\
$\mathrm{K}_{\text {Deep }}{ }^{81}$ & Extended connectivity fingerprint & CNN \\
Pafnucy $^{82}$ & 3D voxel representation & CNN \\
RF::VinaElem & 3D voxel representation & Rntermolecular contacts and Autodock Vina features \\
$\Delta$ vinaRF $_{20}{ }^{58}$ & Autodock Vina and other physical features & Random Forest \\
\hline
\end{tabular}

